\title{
Dual Ramanujan-Fourier series
}

\author{
Noboru Ushiroya
}

\begin{abstract}
Let $c_{q}(n)$ be the Ramanujan sums. Many results concerning Ramanujan-Fourier series $f(n)=\sum_{q=1}^{\infty} a_{q} c_{q}(n)$ are obtained by many mathematicians. In this paper we study series of the form $f(q)=\sum_{n=1}^{\infty} a_{n} c_{q}(n)$, which we call dual RamanujanFourier series. We extend Lucht's theorem and Delange's theorem to this case and obtain some results.
\end{abstract}

Keywords. Ramanujan-Fourier series, Ramanujan sums, arithmetic functions, multiplicative functions.

2010 Mathematics Subject Classification. 11A25, 11N37.

\section{Introduction}

For $q, n \in \mathbb{N}=\{1,2, \cdots\}$, the Ramanujan sums $c_{q}(n)$ are defined in [Ra] by

$$
c_{q}(n)=\sum_{\substack{k=1 \\(k, q)=1}}^{q} \exp \left(\frac{2 \pi i k n}{q}\right),
$$

where $(k, q)$ is the greatest common divisor of $k$ and $q$. Let $f: \mathbb{N} \mapsto \mathbb{C}$ be an arithmetic function. Ramanujan [Ra] investigated its Ramanujan-Fourier series which is an infinite series of the form

$$
f(n)=\sum_{q=1}^{\infty} a_{q} c_{q}(n),
$$

where $a_{q}$ are called the Ramanujan-Fourier coefficients of $f$, and he obtained the following results.

$$
\begin{aligned}
& \frac{\sigma_{s}(n)}{n^{s}}=\zeta(s+1) \sum_{q=1}^{\infty} \frac{c_{q}(n)}{q^{s+1}} \\
& \frac{\varphi_{s}(n)}{n^{s}}=\frac{1}{\zeta(s+1)} \sum_{q=1}^{\infty} \frac{\mu(q)}{\varphi_{s+1}(q)} c_{q}(n), \\
& \tau(n)=-\sum_{q=1}^{\infty} \frac{\log q}{q} c_{q}(n), \\
& r(n)=\pi \sum_{q=1}^{\infty} \frac{(-1)^{q-1}}{2 q-1} c_{2 q-1}(n),
\end{aligned}
$$

where $\sigma_{s}(n)=\sum_{d \mid n} d^{s}$ with $s>0, \quad \zeta(s)$ is the Riemann zeta function, $\varphi_{s}(n)=n^{s} \prod_{p \mid n}\left(1-1 / p^{s}\right)$, $\tau(n)=\sum_{d \mid n} 1, \quad \mu$ is the Möbius function and $r(n)$ is the number of representations of $n$ as the sum of two squares.

Ramanujan [Ra] also investigated dual Ramanujan-Fourier series of the form

$$
f(q)=\sum_{n=1}^{\infty} a_{n} c_{q}(n),
$$

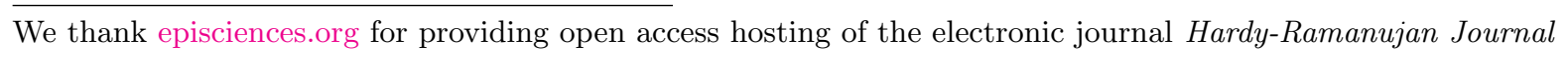


and he obtained the following results.

$$
\begin{aligned}
& \left(\operatorname{id}^{1-s} * \mu\right)(q)=\varphi_{1-s}(q)=\frac{1}{\zeta(s)} \sum_{n=1}^{\infty} \frac{c_{q}(n)}{n^{s}} \quad \text { if } \quad s>1, \\
& \Lambda(q)=-\sum_{n=1}^{\infty} \frac{c_{q}(n)}{n} \quad \text { if } \quad q \geqq 2
\end{aligned}
$$

where id is the function $\operatorname{id}(n)=n, f * g$ denotes the Dirichlet convolution of $f$ and $g$, and $\Lambda(q)$ denotes the von Mangoldt function.

We investigate dual Ramanujan-Fourier series and obtain theorems which are extensions of the results due to Delange and Lucht. Several examples are given. The method used in this paper is quite elementary.

\section{Preliminaries}

Let $\delta(n)=\left\{\begin{array}{lll}1 & \text { if } & n=1 \\ 0 & \text { if } & n>1\end{array}\right.$ and let $\delta(m, n)=\left\{\begin{array}{lll}1 & \text { if } & m=n \\ 0 & \text { if } & m \neq n\end{array}\right.$

We set $D(m, n)=m \delta(m, n)$. Obviously, $D(m, n)=D(n, m)$ holds.

Let $f, g: \mathbb{N} \mapsto \mathbb{C}$ be arithmetic functions. The Dirichlet convolution of $f$ and $g$ is defined by

$$
(f * g)(n)=\sum_{d \mid n} f(d) g(n / d)
$$

For two arithmetic functions, one of which is a function of one variable, the other a function of two variables, we define similar types of convolutions as follows.

Definition 2.1. Let $f: \mathbb{N} \mapsto \mathbb{C}$ be an arithmetic function and $g: \mathbb{N} \times \mathbb{N} \mapsto \mathbb{C}$ be an arithmetic function of two variables. We define $f \underset{\ell}{*} g: \mathbb{N} \times \mathbb{N} \mapsto \mathbb{C}$ and ${ }_{r}^{*} f: \mathbb{N} \times \mathbb{N} \mapsto \mathbb{C}$ as follows.

$$
\begin{aligned}
& (\underset{\ell}{*} g)(q, n)=(f(\cdot) * g(\cdot, n))(q)=\sum_{d \mid q} f\left(\frac{q}{d}\right) g(d, n), \\
& (g \underset{r}{*} f)(q, n)=(g(q, \cdot) * f(\cdot))(n)=\sum_{d \mid n} g(q, d) f\left(\frac{n}{d}\right) .
\end{aligned}
$$

It is clear that the following lemma holds.

Lemma 2.1. Let $f, h: \mathbb{N} \mapsto \mathbb{C}$ be arithmetic functions and let $g: \mathbb{N} \times \mathbb{N} \mapsto \mathbb{C}$ be an arithmetic function of two variables. Then we have

$$
\begin{gathered}
(\underset{\ell}{f} g) \underset{r}{*}=f \underset{\ell}{*}(g \underset{r}{*} h), \\
f \underset{\ell}{*}(h \underset{\ell}{*} g)=(f * h) \underset{\ell}{*} g, \\
(g \underset{r}{*} f) \underset{r}{*}=g \underset{r}{*}(f * h) .
\end{gathered}
$$

We note that $((f \underset{\ell}{*} g) \underset{r}{*} h)(q, n)$ can also be written as $\sum_{d_{1}\left|q, d_{2}\right| n} f\left(q / d_{1}\right) g\left(d_{1}, d_{2}\right) h\left(n / d_{2}\right)$. We simply write $f \underset{\ell}{*} g_{r}^{*} h$ instead of $(f \underset{\ell}{*} g) \underset{r}{*} h$ or $\underset{\ell}{*}\left(g_{r}^{*} h\right)$.

It is easy to see that the following lemma holds. 
Lemma 2.2. Let $f: \mathbb{N} \mapsto \mathbb{C}$ be an arithmetic function. Then we have

$$
\begin{aligned}
& (f \underset{\ell}{*} D)(q, n)=I_{n \mid q} f\left(\frac{q}{n}\right) n=\left\{\begin{array}{ccc}
f\left(\frac{q}{n}\right) n & \text { if } & n \mid q \\
0 & \text { if } & n \nmid q,
\end{array}\right. \\
& \underset{r}{D * f})(q, n)=I_{q \mid n} f\left(\frac{n}{q}\right) q=\left\{\begin{array}{ccc}
f\left(\frac{n}{q}\right) q & \text { if } & q \mid n \\
0 & \text { if } & q \nmid n,
\end{array}\right.
\end{aligned}
$$

where $I_{n \mid q}=\left\{\begin{array}{lll}1 & \text { if } & n \mid q \\ 0 & \text { if } & n \nmid q\end{array}\right.$

Proof. By definiton, we have

$$
(\underset{\ell}{f} \underset{\ell}{*})(q, n)=\sum_{d \mid q} f\left(\frac{q}{d}\right) D(d, n)=\sum_{d \mid q} f\left(\frac{q}{d}\right) n \delta(d, n)=I_{n \mid q} f\left(\frac{q}{n}\right) n .
$$

The proof of the second assertion is similar.

Let $f, g: \mathbb{N} \times \mathbb{N} \mapsto \mathbb{C}$ be arithmetic functions of two variables. The Dirichlet convolution of $f$ and $g$ is defined by

$$
(f * g)(q, n)=\sum_{d_{1}\left|q, d_{2}\right| n} f\left(d_{1}, d_{2}\right) g\left(q / d_{1}, n / d_{2}\right) .
$$

Let $f: \mathbb{N} \mapsto \mathbb{C}$ be an arithmetic function. We note that, if we define $f \otimes \delta, \delta \otimes f: \mathbb{N} \times \mathbb{N} \mapsto \mathbb{C}$ by

$$
\begin{aligned}
& (f \otimes \delta)(q, n)=f(q) \delta(n), \\
& (\delta \otimes f)(q, n)=\delta(q) f(n),
\end{aligned}
$$

then we have for $g: \mathbb{N} \times \mathbb{N} \mapsto \mathbb{C}$

$$
\begin{aligned}
& (f \underset{\ell}{f} g)(q, n)=((f \otimes \delta) * g)(q, n), \\
& (g * f)(q, n)=(g *(\delta \otimes f))(q, n) .
\end{aligned}
$$

We say that $f: \mathbb{N} \mapsto \mathbb{C}$ is a multiplicative function if $f$ satisfies

$$
f\left(n_{1} n_{2}\right)=f\left(n_{1}\right) f\left(n_{2}\right)
$$

for any $n_{1}, n_{2} \in \mathbb{N}$ satisfying $\left(n_{1}, n_{2}\right)=1$. It is well known that if $f$ and $g$ are multiplicative functions, then $f * g$ also becomes a multiplicative function. We say that $f: \mathbb{N} \times \mathbb{N} \mapsto \mathbb{C}$ is a multiplicative function of two variables if $f$ satisfies

$$
f\left(q_{1} q_{2}, n_{1} n_{2}\right)=f\left(q_{1}, n_{1}\right) f\left(q_{2}, n_{2}\right)
$$

for any $q_{1}, q_{2}, n_{1}, n_{2} \in \mathbb{N}$ satisfying $\left(q_{1} n_{1}, q_{2} n_{2}\right)=1$. It is well known that if $f$ and $g$ are multiplicative functions of two variables, then $f * g$ also becomes a multiplicative function of two variables.

It is easy to see that the following lemma holds.

Lemma 2.3. Let $f, h: \mathbb{N} \mapsto \mathbb{C}$ be multiplicative functions and let $g: \mathbb{N} \times \mathbb{N} \mapsto \mathbb{C}$ be a multiplicative function of two variables. Then $f \underset{\ell}{*} g, \quad{ }_{r}^{*} h$ and $\underset{\ell}{*} \underset{r}{g} \underset{r}{*}$ are all multiplicative functions of two variables.

Proof. If $f$ is multiplicative, then $f \otimes \delta$ is also multiplicative as a function of two variables. Therefore $f \underset{\ell}{*} g=(f \otimes \delta) * g$ is also multiplicative as a function of two variables. Similarly, $g \underset{r}{*} h$ and $f \underset{\ell}{*} g_{r} h$ are multiplicative functions of two variables. 
Ramanujan [Ra] proved that $c_{q}(n)$ can be written as

$$
c_{q}(n)=\sum_{d \mid(q, n)} \mu(q / d) d
$$

We show that $c_{q}(n)$ can also be written as follows.

\section{Lemma 2.4 .}

$$
c_{q}(n)=(\underset{\ell}{*} \underset{r}{*} \mathbf{1})(q, n)
$$

where $\mathbf{1}(n)=1$ for every $n \in \mathbb{N}$.

Proof. By definition, we have

$$
(\mu \underset{\ell}{*} D \underset{r}{*} \mathbf{1})(q, n)=\sum_{d_{1}\left|q, d_{2}\right| n} \mu\left(q / d_{1}\right) d_{1} \delta\left(d_{1}, d_{2}\right) \mathbf{1}\left(n / d_{2}\right)=\sum_{d \mid(q, n)} \mu(q / d) d .
$$

Hardy [Ha] proved that, for fixed $n, q \mapsto c_{q}(n)$ is a multiplicative function. Johnson [Jo] proved that $(q, n) \mapsto c_{q}(n)$ is a multiplicative function of two variables. We remark that the multiplicativity of $(q, n) \mapsto c_{q}(n)$ is trivial from Lemma 2.4 and Lemma 2.3 since $D: \mathbb{N} \times \mathbb{N} \mapsto \mathbb{C}$ is multiplicative as a function of two variables.

It is well known that the following holds ([Si]). For a fixed integer $k$,

$$
\sum_{q \mid k} c_{q}(n)=I_{k \mid n} k=\left\{\begin{array}{lll}
k & \text { if } & k \mid n \\
0 & \text { if } & k \nmid n .
\end{array}\right.
$$

We give another expression of the above in the following lemma. We simply write $c$ instead of $c .(\cdot)$. We note that $\sum_{q \mid k} c_{q}(n)$ can be written as $(\underset{\ell}{*} c)(k, n)$.

Lemma 2.5. We have

$$
\begin{aligned}
& \underset{\ell}{\left(\mathbf{1}_{\ell}^{*}\right)(q, n)}=q I_{q \mid n} \quad=\left\{\begin{array}{lll}
q & \text { if } & q \mid n \\
0 & \text { if } & q \nmid n,
\end{array}\right. \text { and } \\
& \underset{r}{(c * \mu)(q, n)}=n I_{n \mid q} \mu\left(\frac{q}{n}\right)=\left\{\begin{array}{lll}
n \mu\left(\frac{q}{n}\right) & \text { if } & n \mid q \\
0 & \text { if } & n \nmid q .
\end{array}\right.
\end{aligned}
$$

Proof. Since $c=\mu \underset{\ell}{*} \underset{r}{*} \mathbf{1}$ and $\mathbf{1} * \mu=\delta$, we have by Lemma 2.2

$$
\begin{aligned}
& (\underset{\ell}{\mathbf{1}} * c)(q, n)=(\underset{\ell}{\mathbf{1}}(\mu \underset{\ell}{*} \underset{r}{D} \mathbf{1}))(q, n)=((\mathbf{1} * \mu) \underset{\ell}{*}(\underset{r}{*} \mathbf{1}))(q, n)=(D \underset{r}{*} \mathbf{1})(q, n)=q I_{q \mid n}, \quad \text { and } \\
& (c \underset{r}{*} \mu)(q, n)=((\mu \underset{\ell}{*} \underset{r}{*} \mathbf{1}) \underset{r}{*} \mu)(q, n)=((\mu \underset{\ell}{*} D) \underset{r}{*}(\mathbf{1} * \mu))(q, n)=(\mu \underset{\ell}{*} D)(q, n)=n I_{n \mid q} \mu\left(\frac{q}{n}\right) \text {. }
\end{aligned}
$$

\section{Some Results}

In this section we show some results concerning dual Ramanujan-Fourier series. First, we introduce the following Lucht's theorem concerning Ramanujan-Fourier series. 
Theorem 3.1. (Lucht $[\mathrm{Lu}]$ ) Let $a: \mathbb{N} \mapsto \mathbb{C}$ be an arithmetic function. If the series

$$
A(n):=n \sum_{k=1}^{\infty} \mu(k) a(k n)
$$

converges for every $n \in \mathbb{N}$, then for $f(n)=(A * \mathbf{1})(n)$, we have

$$
f(n)=\sum_{q=1}^{\infty} a(q) c_{q}(n) .
$$

Lucht obtained (1.4) by taking $a(n)=-\frac{\log n}{n}$. In this case, we see that

$$
A(n)=n \sum_{k=1}^{\infty} \mu(k) a(k n)=-n \sum_{k=1}^{\infty} \mu(k) \frac{\log k+\log n}{k n}=\mathbf{1}(n),
$$

since $\sum_{k=1}^{\infty} \frac{\mu(k) \log k}{k}=-1$ and $\sum_{k=1}^{\infty} \frac{\mu(k)}{k}=0$. Therefore $f=A * \mathbf{1}=\mathbf{1} * \mathbf{1}=\tau$ satisfies (1.4). We would like to extend Lucht's theorem to the case of dual Ramanujan-Fourier series. We show the following theorem which is "dual" to Lucht's theorem.

Theorem 3.2. Let $a: \mathbb{N} \mapsto \mathbb{C}$ be an arithmetic function. If the series

$$
A(q):=q \sum_{k=1}^{\infty} a(k q)
$$

converges for every $q \in \mathbb{N}$, then for $f(q)=(A * \mu)(q)$, we have

$$
f(q)=\sum_{n=1}^{\infty} a(n) c_{q}(n) .
$$

Proof. Since $c_{q}(n)=(\underset{\ell}{*} \underset{r}{*} \mathbf{1})(q, n)$, we have by Lemma 2.2

$$
\begin{aligned}
\sum_{n \leqq x} a(n) c_{q}(n) & =\sum_{n \leqq x} a(n)((\mu \underset{\ell}{*} D) \underset{r}{*} \mathbf{1})(q, n)=\sum_{n \leqq x} a(n) \sum_{d \mid n}(\mu \underset{\ell}{*} D)(q, d) \mathbf{1}\left(\frac{n}{d}\right) \\
& =\sum_{d k \leqq x} a(d k)(\underset{\ell}{*} D)(q, d)=\sum_{d k \leqq x} a(d k) I_{d \mid q} \mu\left(\frac{q}{d}\right) d \\
& =\sum_{d \leqq x} I_{d \mid q} \mu\left(\frac{q}{d}\right) d \sum_{k \leqq x / d} a(d k)=\sum_{d \mid q} \mu\left(\frac{q}{d}\right)\left(d \sum_{k \leqq x / d} a(d k)\right)
\end{aligned}
$$

where $x$ is a sufficiently large real number. Letting $x \rightarrow \infty$, we have

$$
\sum_{n=1}^{\infty} a(n) c_{q}(n)=\sum_{d \mid q} \mu\left(\frac{q}{d}\right) A(d)=(\mu * A)(q)=f(q),
$$

which proves Theorem 3.2.

Remark 3.1. It is easy to see that, if we set $a(n)=1 / n^{s} \quad(s>1)$, then we obtain (1.6) since

$$
A(q)=q \sum_{k=1}^{\infty} a(k q)=q \sum_{k=1}^{\infty} \frac{1}{(k q)^{s}}=\zeta(s) \frac{1}{q^{s-1}}=\zeta(s) \operatorname{id}^{1-s}(q) .
$$


We show other examples of Theorem 3.2 below.

Example 3.1. Let $\omega(q)$ denote the number of distinct prime divisors of $q$ and let $\lambda(q)=(-1)^{\Omega(q)}$ be the Liouville function where $\Omega(q)$ is the number of prime factors of $q$, counted with multiplicity. Then we have

$$
2^{\omega(q)} \lambda(q)=-\frac{1}{\zeta(2)} \sum_{n=1}^{\infty} \frac{\lambda(n) \log n}{n} c_{q}(n) .
$$

Proof. Let $a(n)=\lambda(n) \log n / n$. Then, noting that $\lambda$ is completely multiplicative, we have

$$
\begin{aligned}
A(q) & =q \sum_{k=1}^{\infty} a(k q)=q \sum_{k=1}^{\infty} \frac{\lambda(k q)(\log k+\log q)}{k q} \\
& =\lambda(q) \sum_{k=1}^{\infty} \frac{\lambda(k)(\log k+\log q)}{k}=-\zeta(2) \lambda(q),
\end{aligned}
$$

since $\sum_{k=1}^{\infty} \frac{\lambda(k) \log k}{k}=-\zeta(2)$ and $\sum_{k=1}^{\infty} \frac{\lambda(k)}{k}=0$. Therefore, if we set

$$
f(q)=(A * \mu)(q)=-\zeta(2)(\lambda * \mu)(q)=-\zeta(2) 2^{\omega(q)} \lambda(q),
$$

then Theorem 3.2 gives the desired result.

Example 3.2. Let $s>1$. Then we have

$$
\frac{1}{\zeta(s)}\left(\frac{(\mathrm{id}) \mu}{\varphi_{s}} * \mu\right)(q)=\sum_{n=1}^{\infty} \frac{\mu(n)}{n^{s}} c_{q}(n) .
$$

Proof. Setting $a(n)=\mu(n) / n^{s}$, we have

$$
\begin{aligned}
A(q) & =q \sum_{k=1}^{\infty} a(k q)=q \sum_{k=1}^{\infty} \frac{\mu(k q)}{(k q)^{s}}=q \sum_{\substack{k \geq 1 \\
(k, q)=1}} \frac{\mu(k) \mu(q)}{k^{s} q^{s}} \\
& =\frac{q \mu(q)}{q^{s}} \prod_{p \nmid q}\left(1-\frac{1}{p^{s}}\right)=\frac{q \mu(q)}{q^{s}} \frac{1}{\zeta(s) \prod_{p \mid q}\left(1-1 / p^{s}\right)} \\
& =\frac{1}{\zeta(s)} \frac{\operatorname{id}(q) \mu(q)}{\varphi_{s}(q)} .
\end{aligned}
$$

Theorefore if we set $f=A * \mu=\frac{1}{\zeta(s)} \frac{(\mathrm{id}) \mu}{\varphi_{s}} * \mu$, then we see that (3.10) holds by Theorem 3.2.

Ramanujan $[\mathrm{Ra}]$ proved

$$
\sum_{q=1}^{\infty} \frac{c_{q}(n)}{q}=0,
$$

which is well known to be equivalent to the prime number theorem. We remark that, letting $s \rightarrow 1$ in (3.10), we obtain

$$
\sum_{n=1}^{\infty} \frac{\mu(n) c_{q}(n)}{n}=0
$$

which is "dual" to (3.11).

We also have another example which is "dual" to (3.11). 
Example 3.3. We have

$$
\sum_{n=1}^{\infty} \frac{\lambda(n) c_{q}(n)}{n}=0
$$

Proof. Taking $a(n)=\lambda(n) / n$ in Theorem 3.2, we have

$$
A(q)=q \sum_{k=1}^{\infty} a(k q)=q \sum_{k=1}^{\infty} \frac{\lambda(k q)}{k q}=\lambda(q) \sum_{k=1}^{\infty} \frac{\lambda(k)}{k}=0,
$$

from which $f=A * \mu=0$ follows.

Next we introduce Delange's theorem concerning Ramanujan-Fourier series. Given an arithmetic function $a: \mathbb{N} \mapsto \mathbb{C}$, it is convenient to use Theorem 3.2 in order to find $f$ satisfying (3.9). However, given $f$, it is not convenient to use Theorem 3.2 in order to find $a$ satisfying (3.9). In the case of Ramanujan-Fourier series, it is sometimes useful to use the following Delange's theorem in order to find $a$ satisfying (1.1) for given $f$. We will extend Delange's theorem to the case of dual RamanujanFourier series later.

Theorem 3.3. (Delange [De]) Let $f(n)$ be an arithmetic function satisfying

$$
\sum_{n=1}^{\infty} 2^{\omega(n)} \frac{|(f * \mu)(n)|}{n}<\infty .
$$

Then its Ramanujan-Fourier series is pointwise convergent and

$$
f(n)=\sum_{q=1}^{\infty} a(q) c_{q}(n)
$$

holds where

$$
a(q)=\sum_{m=1}^{\infty} \frac{(f * \mu)(q m)}{q m} .
$$

Moreover, if $f$ is a multiplicative function, then $a(q)$ can be rewritten as

$$
a(q)=\prod_{p \in \mathcal{P}}\left(\sum_{e=\nu_{p}(q)}^{\infty} \frac{(f * \mu)\left(p^{e}\right)}{p^{e}}\right),
$$

where $\mathcal{P}$ denotes the set of prime numbers and $\nu_{p}(q)=\left\{\begin{array}{lll}\alpha & \text { if } & p^{\alpha} \| q \\ 0 & \text { if } & p \nmid q .\end{array}\right.$

Lucht $[\mathrm{Lu}]$ showed that Theorem 3.3 can easily be obtained from Theorem 3.1. We would like to extend Theorem 3.3 to the case of dual Ramanujan-Fourier series by using Theorem 3.2.

Theorem 3.4. Let $f$ be an arithmetic function satisfying

$$
\sum_{q=1}^{\infty} \frac{|(f * \mathbf{1})(q)|}{q} \tau(q)<\infty .
$$

Then its dual Ramanujan-Fourier series is pointwise convergent and

$$
f(q)=\sum_{n=1}^{\infty} a(n) c_{q}(n)
$$

holds where

$$
a(n)=\sum_{m=1}^{\infty} \frac{(f * \mathbf{1})(n m)}{n m} \mu(m) .
$$


Remark 3.2. If $f$ is a multiplicative function satisfying

$$
\sum_{p \in \mathcal{P}} \sum_{e=1}^{\infty} \frac{\left|(f * \mathbf{1})\left(p^{e}\right)\right|}{p^{e}}(e+1)<\infty
$$

then its dual Ramanujan-Fourier series is pointwise convergent and

$$
f(q)=\sum_{n=1}^{\infty} a(n) c_{q}(n)
$$

holds where

$$
a(n)=\sum_{m=1}^{\infty} \frac{(f * \mathbf{1})(n m)}{n m} \mu(m)
$$

Moreover, $a(n)$ can be rewritten as

$$
a(n)=\prod_{p \in \mathcal{P}}\left(\frac{(f * \mathbf{1})\left(p^{\nu_{p}(n)}\right)}{p^{\nu_{p}(n)}}-\frac{(f * \mathbf{1})\left(p^{\nu_{p}(n)+1}\right)}{p^{\nu_{p}(n)+1}}\right) .
$$

Proof of Theorem 3.4. We first see that $A(q)=q \sum_{k=1}^{\infty} a(k q)$ converges since

$$
\begin{aligned}
\sum_{k \leqq x}|a(k q)| & \leqq \sum_{k \leqq x} \sum_{m=1}^{\infty} \frac{|(f * \mathbf{1})(k q m)|}{k q m}|\mu(m)| \\
& \leqq \sum_{\ell=1}^{\infty} \frac{|(f * \mathbf{1})(\ell)|}{\ell} \sum_{k \leqq x, k \mid \ell} 1 \leqq \sum_{\ell=1}^{\infty} \frac{|(f * \mathbf{1})(\ell)|}{\ell} \tau(\ell)<\infty
\end{aligned}
$$

holds for every $x>1$. Using Lemma 2.5 we can rewrite $A(q)$ as follows.

$$
A(q)=q \sum_{k=1}^{\infty} a(k q)=\sum_{m=1}^{\infty} a(m) q I_{q \mid m}=\sum_{m=1}^{\infty} a(m)\left(\mathbf{1}_{\ell}^{* c} c\right)(q, m) .
$$

From this we have

$$
\begin{aligned}
f(q) & \left.=(\mu * A)(q)=\sum_{m=1}^{\infty} a(m)(\underset{\ell}{\mu} \underset{\ell}{*} \underset{\ell}{\mathbf{1}})\right)(q, m)=\sum_{m=1}^{\infty} a(m)((\mu * \mathbf{1}) \underset{\ell}{* c})(q, m) \\
& \left.=\sum_{m=1}^{\infty} a(m) \underset{\ell}{(\delta} c\right)(q, m)=\sum_{m=1}^{\infty} a(m) c_{q}(m) .
\end{aligned}
$$

which completes the proof of Theorem 3.4.

Proof of Remark 3.2. If $f$ is a multiplicative function, then $q \mapsto \frac{(f * \mathbf{1})(q)}{q} \tau(q)$ is also a multiplicative function. Using $1+x \leqq \exp (x)$, we see that (3.16) follows from (3.18) since

$$
\begin{aligned}
\sum_{q \leqq Q} \frac{|(f * \mathbf{1})(q)|}{q} \tau(q) & \leqq \prod_{p \in \mathcal{P}}\left(1+\sum_{e=1}^{\infty} \frac{\left|(f * \mathbf{1})\left(p^{e}\right)\right|}{p^{e}} \tau\left(p^{e}\right)\right) \\
& \leqq \prod_{p \in \mathcal{P}} \exp \left(\sum_{e=1}^{\infty} \frac{\left|(f * \mathbf{1})\left(p^{e}\right)\right|}{p^{e}} \tau\left(p^{e}\right)\right)=\exp \left(\sum_{p \in \mathcal{P}} \sum_{e=1}^{\infty} \frac{\left|(f * \mathbf{1})\left(p^{e}\right)\right|}{p^{e}}(e+1)\right)<\infty
\end{aligned}
$$


holds for every $Q>1$. Therefore (3.17) holds by Theorem 3.4. In the expression of (3.17), we set $n=\prod p_{j}^{e_{j}}, m=r \prod p_{j}^{d_{j}}$ where $(r, n)=1, e_{j} \geqq 1$, and $d_{j} \geqq 0$. Then we have

$$
a(n)=\sum_{d_{j} \geqq 0, r \geqq 1,(r, n)=1} \frac{(f * \mathbf{1})\left(r \prod p_{j}^{e_{j}+d_{j}}\right)}{r \prod p_{j}^{e_{j}+d_{j}}} \mu\left(r \prod p_{j}^{d_{j}}\right) .
$$

Since $f * 1$ is multiplicative and since $\mu\left(p_{j}^{d_{j}}\right)=0$ if $d_{j} \geqq 2$ for some $j$, we obtain

$$
\begin{aligned}
a(n) & =\prod_{j}\left(\sum_{0 \leqq d_{j} \leqq 1} \frac{(f * \mathbf{1})\left(p_{j}^{e_{j}+d_{j}}\right)}{p_{j}^{e_{j}+d_{j}}} \mu\left(p_{j}^{d_{j}}\right)\right) \times \sum_{r \geqq 1,(r, n)=1} \frac{(f * \mathbf{1})(r)}{r} \mu(r) \\
& =\prod_{p \mid n}\left(\frac{(f * \mathbf{1})\left(p^{\nu_{p}(n)}\right)}{p^{\nu_{p}(n)}}-\frac{(f * \mathbf{1})\left(p^{\nu_{p}(n)+1}\right)}{p^{\nu_{p}(n)+1}}\right) \times \prod_{p \nmid n}\left(1-\frac{(f * \mathbf{1})(p)}{p}\right) \\
& =\prod_{p \in \mathcal{P}}\left(\frac{(f * \mathbf{1})\left(p^{\nu_{p}(n)}\right)}{p^{\nu_{p}(n)}}-\frac{(f * \mathbf{1})\left(p^{\nu_{p}(n)+1}\right)}{p^{\nu_{p}(n)+1}}\right),
\end{aligned}
$$

which completes the proof of Remark 3.2.

Several examples are shown below.

Example 3.4. Let $s>1$. Then we have

$$
\frac{\varphi_{s}(q)}{q^{s}} \mu(q)=\frac{1}{\zeta(s+1)} \sum_{n=1}^{\infty} \frac{\varphi(K(n))}{n \psi_{s+1}(K(n))} c_{q}(n),
$$

where $K(n)=\prod_{p \mid n} p$ and $\psi_{s}(n)=n^{s} \prod_{p \mid n}\left(1+1 / p^{s}\right)$.

Proof. Let $f(q)=\frac{\varphi_{s}(q)}{q^{s}} \mu(q)$. Then it is easy to see that

$$
f\left(p^{e}\right)=\left\{\begin{array}{cll}
-\left(1-1 / p^{s}\right) & \text { if } & e=1 \\
0 & \text { if } & e \geqq 2
\end{array}\right.
$$

and

$$
(f * \mathbf{1})\left(p^{e}\right)=\left\{\begin{array}{ccc}
1 & \text { if } & e=0 \\
1 / p^{s} & \text { if } e \geqq 1
\end{array}\right.
$$

from which we see that (3.18) holds. It is also easy to see that

$$
\frac{(f * \mathbf{1})\left(p^{e}\right)}{p^{e}}-\frac{(f * \mathbf{1})\left(p^{e+1}\right)}{p^{e+1}}=\left\{\begin{array}{cl}
1-1 / p^{s+1} & \text { if } e=0 \\
\frac{1}{p^{e+s}}(1-1 / p) & \text { if } e \geqq 1 .
\end{array}\right.
$$

From this and (3.19) we have

$$
\begin{aligned}
a(n) & =\prod_{p \mid n}\left(\frac{(f * \mathbf{1})\left(p^{\nu_{p}(n)}\right)}{p^{\nu_{p}(n)}}-\frac{(f * \mathbf{1})\left(p^{\nu_{p}(n)+1}\right)}{p^{\nu_{p}(n)+1}}\right) \prod_{p \nmid n}\left(\frac{(f * \mathbf{1})\left(p^{\nu_{p}(n)}\right)}{p^{\nu_{p}(n)}}-\frac{(f * \mathbf{1})\left(p^{\nu_{p}(n)+1}\right)}{p^{\nu_{p}(n)+1}}\right) \\
& =\prod_{p \mid n} \frac{1}{p^{\nu_{p}(n)+s}}(1-1 / p) \prod_{p \nmid n}\left(1-1 / p^{s+1}\right) \\
& =\prod_{p \mid n} \frac{1}{p^{\nu_{p}(n)+s}} \frac{1-1 / p}{1-1 / p^{s+1}} \prod_{p \in \mathcal{P}}\left(1-1 / p^{s+1}\right) \\
& =\frac{1}{\zeta(s+1)} \prod_{p \mid n} \frac{1}{p^{\nu_{p}(n)}} \frac{p(1-1 / p)}{p^{s+1}\left(1-1 / p^{s+1}\right)}=\frac{1}{\zeta(s+1)} \frac{\varphi(K(n))}{n \psi_{s+1}(K(n))} .
\end{aligned}
$$


Example 3.5. Let $s>1$. Then we have

$$
\frac{\sigma_{s}(q)}{q^{s}} \mu(q)=\frac{\zeta(s+1)}{\zeta(2 s+2)} \sum_{n=1}^{\infty} \frac{(-1)^{\omega(n)} \varphi(K(n))}{n \psi_{s+1}(K(n))} c_{q}(n) .
$$

Proof. Let $f(q)=\frac{\sigma_{s}(q)}{q^{s}} \mu(q)$. Then it is easy to see that

$$
f\left(p^{e}\right)=\left\{\begin{array}{cl}
-1-1 / p^{s} & \text { if } \quad e=1 \\
0 & \text { if } e \geqq 2
\end{array}\right.
$$

and

$$
(f * \mathbf{1})\left(p^{e}\right)=\left\{\begin{array}{cl}
1 & \text { if } e=0 \\
-1 / p^{s} & \text { if } e \geqq 1,
\end{array}\right.
$$

from which we see that (3.18) holds. It is also easy to see that

$$
\frac{(f * \mathbf{1})\left(p^{e}\right)}{p^{e}}-\frac{(f * \mathbf{1})\left(p^{e+1}\right)}{p^{e+1}}=\left\{\begin{array}{cl}
1+1 / p^{s+1} & \text { if } e=0 \\
\frac{-1}{p^{e+s}}(1-1 / p) & \text { if } e \geqq 1
\end{array}\right.
$$

Therefore by (3.19) we have

$$
\begin{aligned}
a(n) & =\prod_{p \mid n}\left(\frac{(f * \mathbf{1})\left(p^{\nu_{p}(n)}\right)}{p^{\nu_{p}(n)}}-\frac{(f * \mathbf{1})\left(p^{\nu_{p}(n)+1}\right)}{p^{\nu_{p}(n)+1}}\right) \prod_{p \nmid n}\left(\frac{(f * \mathbf{1})\left(p^{\nu_{p}(n)}\right)}{p^{\nu_{p}(n)}}-\frac{(f * \mathbf{1})\left(p^{\nu_{p}(n)+1}\right)}{p^{\nu_{p}(n)+1}}\right) \\
& =\prod_{p \mid n} \frac{-1}{p^{\nu_{p}(n)+s}}(1-1 / p) \prod_{p \nmid n}\left(1+1 / p^{s+1}\right) \\
& =\prod_{p \mid n} \frac{-1}{p^{\nu_{p}(n)+s}} \frac{1-1 / p}{1+1 / p^{s+1}} \prod_{p \in \mathcal{P}}\left(1+1 / p^{s+1}\right) \\
& =\frac{\zeta(s+1)}{\zeta(2 s+2)} \prod_{p \mid n} \frac{-1}{p^{\nu_{p}(n)}} \frac{p(1-1 / p)}{p^{s+1}\left(1+1 / p^{s+1}\right)}=\frac{\zeta(s+1)}{\zeta(2 s+2)} \frac{(-1)^{\omega(n)} \varphi(K(n))}{n \psi_{s+1}(K(n))} .
\end{aligned}
$$

Example 3.6. We have

$$
\lambda(q)=\sum_{n=1}^{\infty} a(n) c_{q}(n)
$$

where $a(n)=\frac{1}{n} \prod_{\substack{p \mid n \\ \nu_{p}(n): \text { odd }}} \frac{-1}{p}$

Proof. Let $f(q)=\lambda(q)$. Then it is easy to see that

$$
(f * \mathbf{1})\left(p^{e}\right)=\left\{\begin{array}{lll}
0 & \text { if } & e \text { is odd } \\
1 & \text { if } & e \text { is even }
\end{array}\right.
$$

from which we see that (3.18) holds. It is also easy to see that

$$
\frac{(f * \mathbf{1})\left(p^{e}\right)}{p^{e}}-\frac{(f * \mathbf{1})\left(p^{e+1}\right)}{p^{e+1}}= \begin{cases}-1 / p^{e+1} & \text { if } e \text { is odd } \\ 1 / p^{e} & \text { if } e \text { is even. }\end{cases}
$$


Therefore by (3.19) we have

$$
\begin{aligned}
a(n)= & \prod_{p \mid n}\left(\frac{(f * \mathbf{1})\left(p^{\nu_{p}(n)}\right)}{p^{\nu_{p}(n)}}-\frac{(f * \mathbf{1})\left(p^{\nu_{p}(n)+1}\right)}{p^{\nu_{p}(n)+1}}\right) \prod_{p \nmid n}\left(\frac{(f * \mathbf{1})\left(p^{\nu_{p}(n)}\right)}{p^{\nu_{p}(n)}}-\frac{(f * \mathbf{1})\left(p^{\nu_{p}(n)+1}\right)}{p^{\nu_{p}(n)+1}}\right) \\
= & \prod_{\substack{p \mid n \\
\nu_{p}(n): \text { odd }}} \frac{-1}{p^{\nu_{p}(n)+1}} \prod_{\substack{p \mid n \\
\nu_{p}(n): \text { even }}} \frac{1}{p^{\nu_{p}(n)}} \prod_{p \nmid n} 1 \\
= & \prod_{p \mid n} \frac{1}{p^{\nu_{p}(n)}} \prod_{\substack{p \mid n \\
\nu_{p}(n): \text { odd }}} \frac{-1}{p}=\frac{1}{n} \prod_{\substack{p \mid n \\
\nu_{p}(n): \text { odd }}} \frac{-1}{p} .
\end{aligned}
$$

\section{Example 3.7.}

$$
\frac{\varphi(q)}{q} \lambda(q)=\frac{1}{\zeta(2)} \sum_{n=1}^{\infty} \frac{I_{\text {square }}(n)}{n} c_{q}(n),
$$

where $I_{\text {square }}(n)= \begin{cases}1 & \text { if } n \text { is a perfect square } \\ 0 & \text { otherwise }\end{cases}$

Proof. Let $f(q)=\frac{\varphi(q)}{q} \lambda(q)$. Then it is easy to see that

$$
(f * \mathbf{1})\left(p^{e}\right)=\left\{\begin{array}{cll}
1 / p & \text { if } & e \text { is odd } \\
1 & \text { if } & e \text { is even }
\end{array}\right.
$$

from which we see that (3.18) holds. It is also easy to see that

$$
\frac{(f * \mathbf{1})\left(p^{e}\right)}{p^{e}}-\frac{(f * \mathbf{1})\left(p^{e+1}\right)}{p^{e+1}}=\left\{\begin{array}{cl}
0 & \text { if } e \text { is odd } \\
\frac{1}{p^{e}}\left(1-1 / p^{2}\right) & \text { if } e \text { is even }
\end{array}\right.
$$

Therefore, by (3.19), $a(n)=0$ if $n$ is not a perfect square. If $n$ is a perfect square, then we have

$$
a(n)=\prod_{p \mid n} \frac{1}{p^{\nu p(n)}}\left(1-1 / p^{2}\right) \prod_{p \nmid n}\left(1-1 / p^{2}\right)=\prod_{p \in \mathcal{P}}\left(1-1 / p^{2}\right) \prod_{p \mid n} \frac{1}{p^{\nu_{p}(n)}}=\frac{1}{\zeta(2)} \frac{1}{n} .
$$

Thus we can express $a(n)$ as

$$
a(n)=\frac{1}{\zeta(2)} \frac{I_{\text {square }}(n)}{n}
$$

whether $n$ is a perfect square or not. This completes the proof of Example 3.7.

Let $\mathcal{F}$ be the set of real valued arithmetic functions and let $\mathcal{A}=\left\{a \in \mathcal{F}: \sum_{q} a(q) c_{q}(n)\right.$ converges. $\}$, $\mathcal{B}=\left\{b \in \mathcal{F}: \sum_{n} b(n) c_{q}(n)\right.$ converges. $\}$. If we define $T: \mathcal{A} \mapsto \mathcal{F}$ and $T^{*}: \mathcal{B} \mapsto \mathcal{F}$ by

$$
\begin{aligned}
& (T a)(n)=\sum_{q} a(q) c_{q}(n), \\
& \left(T^{*} b\right)(q)=\sum_{n} b(n) c_{q}(n),
\end{aligned}
$$

respectively, then we have "formally"

$$
<b, T a>=<T^{*} b, a>
$$

where $\langle b, a\rangle:=\sum_{n} b(n) a(n)$ is an inner product of $a$ and $b$. More precisely, we have the following trivial proposition. 
Proposition 3.1. If $f(n)=\sum_{q} a(q) c_{q}(n)$ and $g(q)=\sum_{n} b(n) c_{q}(n)$. If

$$
\sum_{q, n}\left|a(q) c_{q}(n) b(n)\right|<\infty
$$

then

$$
\sum_{n} f(n) b(n)=\sum_{q} a(q) g(q)
$$

Proof.

$$
\sum_{n} f(n) b(n)=\sum_{q, n} a(q) c_{q}(n) b(n)=\sum_{q} a(q) g(q) .
$$

As an example of the above proposition, we show the following example.

\section{Example 3.8.}

$$
\sum_{n=1}^{\infty} \frac{\varphi(n) I_{n: \text { square }}}{n^{2}}=\sum_{q=1}^{\infty} \frac{\mu(q)^{2}}{q \psi(q)} .
$$

Proof. By (1.3) with $s=1$ and Example 3.7, we have

$$
\begin{gathered}
\frac{\varphi(n)}{n}=\frac{1}{\zeta(2)} \sum_{q=1}^{\infty} \frac{\mu(q)}{\varphi_{2}(q)} c_{q}(n), \\
\frac{\varphi(q)}{q} \lambda(q)=\frac{1}{\zeta(2)} \sum_{n=1}^{\infty} \frac{I_{\text {square }}(n)}{n} c_{q}(n) .
\end{gathered}
$$

We note that the right hand of (1.3) is absolutely convergent. Hence (3.20) holds. By Proposition 3.20 we have

$$
\begin{aligned}
\sum_{n=1}^{\infty} \frac{\varphi(n)}{n} \frac{I_{n: s q u a r e}}{n} & =\sum_{q=1}^{\infty} \frac{\mu(q)}{\varphi_{2}(q)} \frac{\varphi(q)}{q} \lambda(q)=\sum_{q=1}^{\infty} \frac{\mu(q) \lambda(q) \prod_{p \mid q}(1-1 / p)}{q^{2} \prod_{p \mid q}\left(1-1 / p^{2}\right)} \\
& =\sum_{q=1}^{\infty} \frac{\mu(q)^{2}}{q^{2} \prod_{p \mid q}(1+1 / p)}=\sum_{q=1}^{\infty} \frac{\mu(q)^{2}}{q \psi(q)}
\end{aligned}
$$

which completes the proof of Example 3.8.

Of course, Example 3.8 can also be obtained by expressing both sides as infinite products by prime numbers.

Remark 3.3. We do not know whether we can loosen the condition (3.20) or not. If we can, then, for every $f \in T \mathcal{A}$ such that $f=T a$ and for every $g \in T^{*} \mathcal{B}$ such that $g=T^{*} b$, we have "formally"

$$
\begin{aligned}
& \sum_{n} \frac{f(n) \mu(n)}{n}=<T a, \frac{\mu}{\mathrm{id}}>=<a, T^{*} \frac{\mu}{\mathrm{id}}>=<a, 0>=0, \\
& \sum_{q} \frac{g(q)}{q}=<T^{*} b, \frac{1}{\mathrm{id}}>=<b, T \frac{1}{\mathrm{id}}>=<b, 0>=0,
\end{aligned}
$$

namely, $\operatorname{Im} T \perp \operatorname{Ker} T^{*}$ and $\operatorname{Im} T^{*} \perp \operatorname{Ker} T$. However, we can't prove the above rigorously. 
Next we consider Dirichlet series of a function expressed as Ramanujan-Fourier series or dual Ramanujan-Fourier series. We show the following theorem.

Theorem 3.5. Suppose $s>1$. Let $f$ be an arithmetic function such that the Dirichlet series $\sum_{n=1}^{\infty} \frac{f(n)}{n^{s}}$ converges absolutely and let $a(\cdot)$ be a multiplicative function such that $\sum_{k, n \geqq 1} \frac{|a(k n)|}{n^{s-1}}<\infty$.

(i) If $f(n)=\sum_{q=1}^{\infty} a(q) c_{q}(n)$ converges absolutely, then the Dirichlet series of $f$ is expressed as

$$
\sum_{n=1}^{\infty} \frac{f(n)}{n^{s}}=\zeta(s) \prod_{p \in \mathcal{P}}\left(\sum_{e \geqq 0} \frac{a\left(p^{e}\right)-a\left(p^{e+1}\right)}{p^{e(s-1)}}\right) .
$$

(ii) If $f(q)=\sum_{n=1}^{\infty} a(n) c_{q}(n)$ converges absolutely, then the Dirichlet series of $f$ is expressed as

$$
\sum_{q=1}^{\infty} \frac{f(q)}{q^{s}}=\frac{1}{\zeta(s)} \prod_{p \in \mathcal{P}}\left(\sum_{e_{1} \geqq 0} \frac{\sum_{e_{2} \geqq e_{1}} a\left(p^{e_{2}}\right)}{p^{e_{1}(s-1)}}\right) .
$$

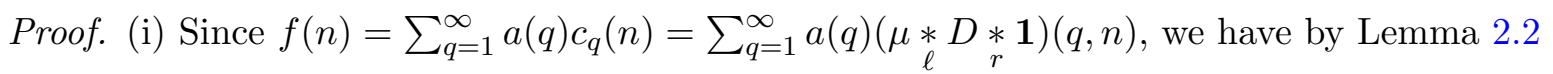

$$
\begin{aligned}
(f * \mu)(n) & =\sum_{q=1}^{\infty} a(q)\left((\mu \underset{\ell}{*} \underset{r}{*} \underset{r}{*} \underset{r}{*} \mu)(q, n)=\sum_{q=1}^{\infty} a(q)(\mu \underset{\ell}{*} D)(q, n)\right. \\
& =\sum_{q=1}^{\infty} a(q) I_{n \mid q} \mu\left(\frac{q}{n}\right) n=\sum_{\substack{q \geqq 1 \\
n \mid q}} a(q) \mu\left(\frac{q}{n}\right) n .
\end{aligned}
$$

From this we have

$$
\begin{aligned}
\sum_{n=1}^{\infty} \frac{(f * \mu)(n)}{n^{s}} & =\sum_{n=1}^{\infty} \frac{1}{n^{s}} \sum_{\substack{q \geqq 1 \\
n \mid q}} a(q) \mu\left(\frac{q}{n}\right) n=\sum_{q \geqq 1} a(q) \sum_{n \mid q} \frac{\mu\left(\frac{q}{n}\right)}{n^{s-1}}=\sum_{q=1}^{\infty} a(q)\left(\frac{1}{\mathrm{id}^{s-1}} * \mu\right)(q) \\
& =\prod_{p \in \mathcal{P}}\left(\sum_{e \geqq 0} a\left(p^{e}\right)\left(\frac{1}{\mathrm{id}^{s-1}} * \mu\right)\left(p^{e}\right)\right)=\prod_{p \in \mathcal{P}}\left(1+\sum_{e \geqq 1} a\left(p^{e}\right)\left(\frac{1}{p^{e(s-1)}}-\frac{1}{p^{(e-1)(s-1)}}\right)\right) \\
& =\prod_{p \in \mathcal{P}}\left(1-a(p)+\sum_{e \geqq 1} \frac{a\left(p^{e}\right)-a\left(p^{e+1}\right)}{p^{e(s-1)}}\right)=\prod_{p \in \mathcal{P}}\left(\sum_{e \geqq 0} \frac{a\left(p^{e}\right)-a\left(p^{e+1}\right)}{p^{e(s-1)}}\right) .
\end{aligned}
$$

Therefore we have

$$
\frac{1}{\zeta(s)} \sum_{n=1}^{\infty} \frac{f(n)}{n^{s}}=\prod_{p \in \mathcal{P}}\left(\sum_{e \geqq 0} \frac{a\left(p^{e}\right)-a\left(p^{e+1}\right)}{p^{e(s-1)}}\right) .
$$

(ii) We proceed in a similar manner. Since $\left.f(q)=\sum_{n=1}^{\infty} a(n) c_{q}(n)=\sum_{n=1}^{\infty} a(n) \underset{\ell}{(\mu \underset{r}{*} D} \underset{\mathbf{1}}{*}\right)(q, n)$, we have by Lemma 2.2

$$
\begin{aligned}
& \left.(\mathbf{1} * f)(q)=\sum_{n=1}^{\infty} a(n)\left(\mathbf{1}_{\ell}^{*} \underset{\ell}{(\mu} \underset{r}{*} \underset{\mathbf{1}}{*}\right)\right)(q, n)=\sum_{n=1}^{\infty} a(n)(D \underset{r}{*} \mathbf{1})(q, n) \\
& =\sum_{n=1}^{\infty} a(n) I_{q \mid n} q=\sum_{\substack{n \geqq 1 \\
q \mid n}} a(n) q .
\end{aligned}
$$


From this we have

$$
\begin{aligned}
\sum_{q=1}^{\infty} \frac{(\mathbf{1} * f)(q)}{q^{s}} & =\sum_{q=1}^{\infty} \frac{1}{q^{s}} \sum_{\substack{n \geqq 1 \\
q \mid n}} a(n) q=\sum_{n \geqq 1} a(n) \sum_{q \mid n} \frac{1}{q^{s-1}} \\
& =\sum_{n=1}^{\infty} a(n)\left(\frac{1}{\mathrm{id}^{s-1}} * \mathbf{1}\right)(n)=\prod_{p \in \mathcal{P}}\left(\sum_{e \geqq 0} a\left(p^{e}\right)\left(\frac{1}{\mathrm{id}^{s-1}} * \mathbf{1}\right)\left(p^{e}\right)\right) \\
& =\prod_{p \in \mathcal{P}}\left(\sum_{e \geqq 0} a\left(p^{e}\right)\left(\mathrm{id}^{1-s} * \mathbf{1}\right)\left(p^{e}\right)\right)=\prod_{p \in \mathcal{P}}\left(\sum_{e_{2} \geqq 0} a\left(p^{e_{2}}\right) \sum_{0 \leqq e_{1} \leqq e_{2}} \frac{1}{p^{e_{1}(s-1)}}\right) \\
& =\prod_{p \in \mathcal{P}}\left(\sum_{e_{1} \geqq 0} \frac{\sum_{e_{2} \geqq e_{1}} a\left(p^{e_{2}}\right)}{p^{e_{1}(s-1)}}\right) .
\end{aligned}
$$

Therefore we have

$$
\zeta(s) \sum_{q=1}^{\infty} \frac{f(q)}{q^{s}}=\prod_{p \in \mathcal{P}}\left(\sum_{e_{1} \geqq 0} \frac{\sum_{e_{2} \geqq e_{1}} a\left(p^{e_{2}}\right)}{p^{e_{1}(s-1)}}\right),
$$

which completes the proof of Theorem 3.5.

As an example of Theorem 3.5, we show the following example.

\section{Example 3.9.}

$$
\frac{\lambda(q) K(q) \psi(q)}{q^{2}}=\frac{\zeta(2)}{\zeta(4)} \sum_{n=1}^{\infty} \frac{\lambda(n)}{n^{2}} c_{q}(n) .
$$

Proof. Let $a(n)=\lambda(n) / n^{2}$. Then we have

$$
\begin{aligned}
& \sum_{e_{2} \geqq e_{1}} a\left(p^{e_{2}}\right)=\frac{(-1)^{e_{1}}}{p^{2 e_{1}}}+\frac{(-1)^{e_{1}+1}}{p^{2\left(e_{1}+1\right)}}+\cdots=\frac{(-1)^{e_{1}}}{p^{2 e_{1}}\left(1+1 / p^{2}\right)}, \quad \text { and } \\
& \sum_{e_{1} \geqq 0} \frac{\sum_{e_{2} \geqq e_{1}} a\left(p^{e_{2}}\right)}{p^{e_{1}(s-1)}}=\frac{1}{1+1 / p^{2}} \sum_{e \geqq 0} \frac{(-1)^{e}}{p^{e(s+1)}}=\frac{1}{1+1 / p^{2}} \frac{1}{1+1 / p^{s+1}} .
\end{aligned}
$$

From this we have

$$
\frac{1}{\zeta(s)} \prod_{p \in \mathcal{P}}\left(\sum_{e_{1} \geqq 0} \frac{\sum_{e_{2} \geqq e_{1}} a\left(p^{e_{2}}\right)}{p^{e_{1}(s-1)}}\right)=\frac{1}{\zeta(s)} \prod_{p \in \mathcal{P}} \frac{1}{1+1 / p^{2}} \frac{1}{1+1 / p^{s+1}}=\frac{1}{\zeta(s)} \frac{\zeta(4)}{\zeta(2)} \frac{\zeta(2 s+2)}{\zeta(s+1)} .
$$

Therefore, if we set $f(q)=\sum_{n=1}^{\infty} a(n) c_{q}(n)$, then $f$ satisfies

$$
\sum_{q=1}^{\infty} \frac{f(q)}{q^{s}}=\frac{1}{\zeta(s)} \frac{\zeta(4)}{\zeta(2)} \frac{\zeta(2 s+2)}{\zeta(s+1)} .
$$

On the other hand, if we set $\widetilde{f}(q)=\frac{\lambda(q) K(q) \psi(q)}{q^{2}}$, then $\widetilde{f}$ satisfies

$$
\begin{aligned}
\sum_{q=1}^{\infty} \frac{\tilde{f}(q)}{q^{s}} & =\prod_{p \in \mathcal{P}}\left(1+\sum_{e \geqq 1} \frac{1}{p^{e s}} \frac{\lambda\left(p^{e}\right) K\left(p^{e}\right) \psi\left(p^{e}\right)}{p^{2 e}}\right)=\prod_{p \in \mathcal{P}}\left(1+\sum_{e \geqq 1} \frac{1}{p^{e s}} \frac{(-1)^{e} p \cdot p^{e}(1+1 / p)}{p^{2 e}}\right) \\
& =\prod_{p \in \mathcal{P}}\left(1+(p+1) \sum_{e \geqq 1}\left(\frac{-1}{p^{e(s+1)}}\right)\right)=\prod_{p \in \mathcal{P}}\left(1-\frac{p+1}{p^{s+1}+1}\right) \\
& =\prod_{p \in \mathcal{P}} \frac{1-1 / p^{s}}{1+1 / p^{s+1}}=\frac{\zeta(2 s+2)}{\zeta(s) \zeta(s+1)} .
\end{aligned}
$$


By the uniqueness of the Dirichlet series, we have $\widetilde{f}(q)=\frac{\zeta(2)}{\zeta(4)} f(q)$, namely

$$
\frac{\lambda(q) K(q) \psi(q)}{q^{2}}=\frac{\zeta(2)}{\zeta(4)} \sum_{n=1}^{\infty} \frac{\lambda(n)}{n^{2}} c_{q}(n) .
$$

\section{The Case of Arithmetic Functions of Two Variables}

In this section, we consider the case of arithmetic functions of two variables. We would like to extend theorems in section 3 to this case. In more detail, we consider Ramanujan-Fourier series

$$
f\left(n_{1}, n_{2}\right)=\sum_{q_{1}, q_{2}=1}^{\infty} a\left(q_{1}, q_{2}\right) c_{q_{1}}\left(n_{1}\right) c_{q_{2}}\left(n_{2}\right),
$$

and dual Ramanujan-Fourier series

$$
f\left(q_{1}, q_{2}\right)=\sum_{n_{1}, n_{2}=1}^{\infty} a\left(n_{1}, n_{2}\right) c_{q_{1}}\left(n_{1}\right) c_{q_{2}}\left(n_{2}\right),
$$

where $f, a$ are arithmetic functions of two variables.

We use the same notations 1 and $\mu$ for the functions

$$
\begin{aligned}
& \mathbf{1}\left(n_{1}, n_{2}\right)=\mathbf{1}\left(n_{1}\right) \mathbf{1}\left(n_{2}\right), \\
& \mu\left(n_{1}, n_{2}\right)=\mu\left(n_{1}\right) \mu\left(n_{2}\right),
\end{aligned}
$$

respectively. Clearly, $(\mu * \mathbf{1})\left(n_{1}, n_{2}\right)=\delta\left(n_{1}\right) \delta\left(n_{2}\right)$ holds.

We begin with the following theorem which is an extension of Theorem 3.1.

Theorem 4.1. Let $a: \mathbb{N} \times \mathbb{N} \mapsto \mathbb{C}$ be an arithmetic function of two variables. If the series

$$
A\left(n_{1}, n_{2}\right):=n_{1} n_{2} \sum_{k_{1}, k_{2}=1}^{\infty} \mu\left(k_{1}, k_{2}\right) a\left(k_{1} n_{1}, k_{2} n_{2}\right)
$$

converges for every $n_{1}, n_{2} \in \mathbb{N}$, then for $f\left(n_{1}, n_{2}\right)=(A * \mathbf{1})\left(n_{1}, n_{2}\right)$, we have

$$
f\left(n_{1}, n_{2}\right)=\sum_{q_{1}, q_{2}=1}^{\infty} a\left(q_{1}, q_{2}\right) c_{q_{1}}\left(n_{1}\right) c_{q_{2}}\left(n_{2}\right) .
$$

Proof. Since $c_{q}(n)=\underset{\ell}{\mu \underset{r}{*} D} \underset{\mathbf{1}}{*}(q, n)$, we have

$$
\begin{aligned}
& \sum_{\substack{q_{1} \leqq \\
q_{2} \leqq}} a\left(q_{1}, q_{2}\right) c_{q_{1}}\left(n_{1}\right) c_{q_{2}}\left(n_{2}\right)=\sum_{\substack{q_{1} \leqq x \\
q_{2} \leqq y}} a\left(q_{1}, q_{2}\right)(\mu \underset{\ell}{\mu} \underset{r}{*} \underset{r}{\mathbf{1}})\left(q_{1}, n_{1}\right)(\underset{\ell}{\mu} \underset{r}{*} \underset{\mathbf{1}}{*})\left(q_{2}, n_{2}\right) \\
& =\sum_{\substack{q_{1} \leqq x \\
q_{2} \leqq y}} a\left(q_{1}, q_{2}\right)\left(\sum_{d_{1} \mid q_{1}} \mu\left(\frac{q_{1}}{d_{1}}\right)(\underset{r}{D} \underset{r}{*})\left(d_{1}, n_{1}\right)\right)\left(\sum_{d_{2} \mid q_{2}} \mu\left(\frac{q_{2}}{d_{2}}\right)(\underset{r}{D * \mathbf{1}})\left(d_{2}, n_{2}\right)\right) .
\end{aligned}
$$


Setting $q_{1}=d_{1} k_{1}, q_{2}=d_{2} k_{2}$ and using Lemma 2.2, we see that the above is equal to

$$
\begin{aligned}
& \sum_{\substack{d_{1} k_{1} \leqq x \\
d_{2} k_{2} \leqq y}} a\left(d_{1} k_{1}, d_{2} k_{2}\right) \mu\left(k_{1}\right) \mu\left(k_{2}\right)(D * \mathbf{1})\left(d_{1}, n_{1}\right)(D * \mathbf{1})\left(d_{2}, n_{2}\right) \\
= & \sum_{\substack{d_{1} k_{1} \leqq x \\
d_{2} k_{2} \leqq y}} a\left(d_{1} k_{1}, d_{2} k_{2}\right) \mu\left(k_{1}\right) \mu\left(k_{2}\right) I_{d_{1} \mid n_{1}} d_{1} I_{d_{2} \mid n_{2}} d_{2} \\
= & \sum_{\substack{d_{1} \leqq x \\
d_{2} \leqq y}} I_{d_{1} \mid n_{1}} I_{d_{2} \mid n_{2}} d_{1} d_{2} \sum_{\substack{k_{1} \leqq x / d_{1} \\
k_{2} \leqq y / d_{2}}} \mu\left(k_{1}, k_{2}\right) a\left(d_{1} k_{1}, d_{2} k_{2}\right),
\end{aligned}
$$

where $x, y$ are sufficiently large real numbers. Letting $x, y \rightarrow \infty$, we have

$$
\begin{aligned}
\sum_{q_{1}, q_{2}=1}^{\infty} a\left(q_{1}, q_{2}\right) c_{q_{1}}\left(n_{1}\right) c_{q_{2}}\left(n_{2}\right) & =\sum_{\substack{d_{1}, d_{2}=1\\
}}^{\infty} I_{d_{1} \mid n_{1}} I_{d_{2} \mid n_{2}}\left(d_{1} d_{2} \sum_{k_{1}, k_{2}=1}^{\infty} \mu\left(k_{1}, k_{2}\right) a\left(d_{1} k_{1}, d_{2} k_{2}\right)\right) \\
& \sum_{\substack{d_{1}\left|n_{1} \\
d_{2}\right| n_{2}}}^{\infty} A\left(d_{1}, d_{2}\right)=(A * \mathbf{1})\left(n_{1}, n_{2}\right)=f\left(n_{1}, n_{2}\right),
\end{aligned}
$$

which proves Theorem 4.1 .

The following theorem is an extension of Theorem 3.2.

Theorem 4.2. Let $a: \mathbb{N} \times \mathbb{N} \mapsto \mathbb{C}$ be an arithmetic function of two variables. If the series

$$
A\left(q_{1}, q_{2}\right):=q_{1} q_{2} \sum_{k_{1}, k_{2}=1}^{\infty} a\left(k_{1} q_{1}, k_{2} q_{2}\right)
$$

converges for every $q_{1}, q_{2} \in \mathbb{N}$, then for $f\left(q_{1}, q_{2}\right)=(A * \mu)\left(q_{1}, q_{2}\right)$, we have

$$
f\left(q_{1}, q_{2}\right)=\sum_{n_{1}, n_{2}=1}^{\infty} a\left(n_{1}, n_{2}\right) c_{q_{1}}\left(n_{1}\right) c_{q_{2}}\left(n_{2}\right) .
$$

Proof. The proof proceeds along the same lines as the proof of Theorem 3.2. We have

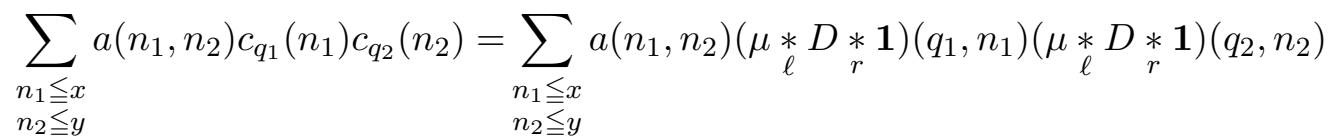

$$
\begin{aligned}
& =\sum_{\substack{n_{1} \leqq x \\
n_{2} \leqq y}} a\left(n_{1}, n_{2}\right)\left(\sum_{d_{1} \mid n_{1}}(\underset{\ell}{*} D)\left(q_{1}, d_{1}\right) \mathbf{1}\left(\frac{n_{1}}{d_{1}}\right)\right)\left(\sum_{d_{2} \mid n_{2}}(\mu \underset{\ell}{*} D)\left(q_{2}, d_{2}\right) \mathbf{1}\left(\frac{n_{2}}{d_{2}}\right)\right) .
\end{aligned}
$$

Setting $n_{1}=d_{1} k_{1}, n_{2}=d_{2} k_{2}$ and using Lemma 2.2, we see that the above is equal to

$$
\begin{aligned}
& \sum_{\substack{d_{1} k_{1} \leqq \\
d_{2} k_{2} \leqq y}} a\left(d_{1} k_{1}, d_{2} k_{2}\right)(\mu * D)\left(q_{1}, d_{1}\right)(\mu * D)\left(q_{2}, d_{2}\right) \\
= & \sum_{\substack{d_{1} k_{1} \leqq x \\
d_{2} k_{2} \leqq y}} a\left(d_{1} k_{1}, d_{2} k_{2}\right) I_{d_{1} \mid q_{1}} \mu\left(\frac{q_{1}}{d_{1}}\right) d_{1} I_{d_{2} \mid q_{2}} \mu\left(\frac{q_{2}}{d_{2}}\right) d_{2} \\
= & \sum_{\substack{d_{1} \leqq x \\
d_{2} \leqq y}} I_{d_{1} \mid q_{1}} I_{d_{2} \mid q_{2}} \mu\left(\frac{q_{1}}{d_{1}}\right) \mu\left(\frac{q_{2}}{d_{2}}\right)\left(d_{1} d_{2} \sum_{\substack{k_{1} \leqq x / d_{1} \\
k_{2} \leqq y / d_{2}}} a\left(d_{1} k_{1}, d_{2} k_{2}\right)\right),
\end{aligned}
$$


where $x, y$ are sufficiently large real numbers. Letting $x, y \rightarrow \infty$, we have

$$
\begin{aligned}
\sum_{n_{1}, n_{2}=1}^{\infty} a\left(n_{1}, n_{2}\right) c_{q_{1}}\left(n_{1}\right) c_{q_{2}}\left(n_{2}\right) & =\sum_{\substack{d_{1}, d_{2}=1 \\
=}}^{\infty} I_{d_{1} \mid q_{1}} I_{d_{2} \mid q_{2}} \mu\left(\frac{q_{1}}{d_{1}}\right) \mu\left(\frac{q_{2}}{d_{2}}\right) A\left(d_{1}, d_{2}\right) \\
& \sum_{\substack{d_{1}\left|q_{1} \\
d_{2}\right| q_{2}}} \mu\left(\frac{q_{1}}{d_{1}}\right) \mu\left(\frac{q_{2}}{d_{2}}\right) A\left(d_{1}, d_{2}\right)=(\mu * A)\left(q_{1}, q_{2}\right)=f\left(q_{1}, q_{2}\right),
\end{aligned}
$$

which proves Theorem 4.2.

The following example is an extension of Example 3.2.

Example 4.1. Let $s>1$. Then we have

$$
\left(\prod_{p \in \mathcal{P}}\left(1-\frac{2}{p^{s}}\right)\right)\left(\frac{q_{1} q_{2} \mu\left(q_{1} q_{2}\right)}{\widetilde{\varphi_{s}}\left(q_{1}\right) \widetilde{\varphi_{s}}\left(q_{2}\right)} * \mu\right)\left(q_{1}, q_{2}\right)=\sum_{n_{1}, n_{2}=1}^{\infty} \frac{\mu\left(n_{1} n_{2}\right)}{\left(n_{1} n_{2}\right)^{s}} c_{q_{1}}\left(n_{1}\right) c_{q_{2}}\left(n_{2}\right),
$$

where $\widetilde{\varphi_{s}}(q)=q^{s} \prod_{p \mid q}\left(1-2 / p^{s}\right)$.

Proof. Setting $a\left(n_{1}, n_{2}\right)=\frac{\mu\left(n_{1} n_{2}\right)}{\left(n_{1} n_{2}\right)^{s}}$ we have

$$
\begin{aligned}
A\left(q_{1}, q_{2}\right) & =q_{1} q_{2} \sum_{k_{1}, k_{2}=1}^{\infty} a\left(k_{1} q_{1}, k_{2} q_{2}\right)=q_{1} q_{2} \sum_{\substack{k_{1}, k_{2}=1 \\
k_{1}}}^{\infty} \frac{\mu\left(k_{1} q_{1} k_{2} q_{2}\right)}{\left(k_{1} q_{1} k_{2} q_{2}\right)^{s}} \\
& =q_{1} q_{2} \sum_{\substack{k_{1}, k_{2} \geqq 1 \\
\left(k_{1} k_{2}, q_{1} q_{2}\right)=1}} \frac{\mu\left(k_{1} k_{2}\right) \mu\left(q_{1} q_{2}\right)}{\left(k_{1} k_{2}\right)^{s}\left(q_{1} q_{2}\right)^{s}}=\frac{\mu\left(q_{1} q_{2}\right)}{\left(q_{1} q_{2}\right)^{s-1}} \sum_{\substack{k \geqq 1 \\
\left(k, q_{1} q_{2}\right)=1}} \frac{\mu(k)}{k^{s}} \sum_{k_{1} \mid k} 1 \\
& =\frac{\mu\left(q_{1} q_{2}\right)}{\left(q_{1} q_{2}\right)^{s-1}} \sum_{\substack{k \geqq 1 \\
\left(k, q_{1} q_{2}\right)=1}} \frac{\mu(k) \tau(k)}{k^{s}}=\frac{\mu\left(q_{1} q_{2}\right)}{\left(q_{1} q_{2}\right)^{s-1}} \prod_{p \nmid q_{1} q_{2}}\left(1+\frac{\mu(p) \tau(p)}{p^{s}}\right) \\
& =\frac{\mu\left(q_{1} q_{2}\right)}{\left(q_{1} q_{2}\right)^{s-1}} \prod_{p \nmid q_{1} q_{2}}\left(1-\frac{2}{p^{s}}\right)=\frac{\mu\left(q_{1} q_{2}\right)}{\left(q_{1} q_{2}\right)^{s-1}} \frac{\prod_{p \in \mathcal{P}}\left(1-2 / p^{s}\right)}{\prod_{p \mid q_{1} q_{2}}\left(1-2 / p^{s}\right)} .
\end{aligned}
$$

If $\left(q_{1}, q_{2}\right)>1$, then $A\left(q_{1}, q_{2}\right)=0$ since $\mu\left(q_{1} q_{2}\right)=0$. If $\left(q_{1}, q_{2}\right)=1$, then we have

$$
\begin{aligned}
A\left(q_{1}, q_{2}\right) & =\frac{\mu\left(q_{1} q_{2}\right)}{\left(q_{1} q_{2}\right)^{s-1}} \frac{\prod_{p \in \mathcal{P}}\left(1-2 / p^{s}\right)}{\prod_{p \mid q_{1}}\left(1-2 / p^{s}\right) \prod_{p \mid q_{2}}\left(1-2 / p^{s}\right)} \\
& =\frac{q_{1} q_{2} \mu\left(q_{1} q_{2}\right)}{\left(q_{1}^{s} \prod_{p \mid q_{1}}\left(1-2 / p^{s}\right)\right)\left(q_{2}^{s} \prod_{p \mid q_{2}}\left(1-2 / p^{s}\right)\right)} \prod_{p \in \mathcal{P}}\left(1-2 / p^{s}\right) \\
& =\frac{q_{1} q_{2} \mu\left(q_{1} q_{2}\right)}{\widetilde{\varphi_{s}}\left(q_{1}\right) \widetilde{\varphi_{s}}\left(q_{2}\right)} \prod_{p \in \mathcal{P}}\left(1-2 / p^{s}\right),
\end{aligned}
$$

which clearly holds also in the case $\left(q_{1}, q_{2}\right)>1$. If we set $f=A * \mu$, then Theorem 4.2 gives the desired result.

Remark 4.1. We consider the case $s \downarrow 1$ in (4.22), where the notation $s \downarrow 1$ means that $s$ approaches 
1 from above. Since

$$
\begin{aligned}
\prod_{p \in \mathcal{P}}\left(1-\frac{2}{p^{s}}\right) & =\left(1-\frac{2}{2^{s}}\right) \prod_{\substack{p \in \mathcal{P} \\
p \geqq 3}}\left(1-\frac{2}{p^{s}}\right)=\left(1-\frac{2}{2^{s}}\right) \prod_{\substack{p \in \mathcal{P} \\
p \geqq 3}}\left(1-2 / p^{s}\right) \frac{\left(1-1 / p^{s}\right)^{2}}{1-2 / p^{s}+1 / p^{2 s}} \\
& =\left(1-\frac{2}{2^{s}}\right) \prod_{\substack{p \in \mathcal{P} \\
p \geqq 3}} \frac{\left(1-1 / p^{s}\right)^{2}}{1-2 / p^{s}+1 / p^{2 s}}=\left(1-\frac{2}{2^{s}}\right) \prod_{\substack{p \in \mathcal{P} \\
p \geqq 3}} \frac{\left(1-1 / p^{s}\right)^{2}}{1+\frac{1}{p^{2 s}\left(1-2 / p^{s}\right)}} \\
& =\left(1-\frac{2}{2^{s}}\right) \prod_{\substack{p \in \mathcal{P} \\
p \geqq 3}}\left(1-\frac{1}{p^{s}}\right)^{2} \prod_{\substack{p \in \mathcal{P} \\
p \geqq 3}} \frac{1}{1+\frac{1}{p^{s}\left(p^{s}-2\right)}} \\
& =\left(1-\frac{2}{2^{s}}\right) \frac{1}{\left(1-\frac{1}{2^{s}}\right)^{2} \zeta^{2}(s)} \prod_{\substack{p \in \mathcal{P} \\
p \geqq 3}} \frac{1}{1+\frac{1}{p^{s}\left(p^{s}-2\right)}}
\end{aligned}
$$

we have

$$
\begin{aligned}
& \lim _{s \downarrow 1}\left(\prod_{p \in \mathcal{P}}\left(1-\frac{2}{p^{s}}\right)\right) \frac{q_{1} q_{2} \mu\left(q_{1} q_{2}\right)}{\widetilde{\varphi_{s}}\left(q_{1}\right) \widetilde{\varphi_{s}}\left(q_{2}\right)} \\
= & \lim _{s \downarrow 1}\left(1-\frac{2}{2^{s}}\right) \frac{1}{\left(1-\frac{1}{2^{s}}\right)^{2} \zeta^{2}(s)}\left(\prod_{p \in \mathcal{P}} \frac{1}{\left.1+\frac{1}{p \geqq 3}\right) \frac{q_{1} q_{2} \mu\left(q_{1} q_{2}\right)}{\widetilde{p_{s}\left(p^{s}-2\right)}\left(q_{1}\right) \widetilde{\varphi_{s}}\left(q_{2}\right)}}\right. \\
= & \lim _{s \downarrow 1}\left(1-\frac{2}{2^{s}}\right) \frac{1}{\zeta^{2}(s)} \frac{q_{1} q_{2} \mu\left(q_{1} q_{2}\right)}{q_{1}^{s} \prod_{p \mid q_{1}}\left(1-2 / p^{s}\right) q_{2}^{s} \prod_{p \mid q_{2}}\left(1-2 / p^{s}\right)} \frac{1}{\left(1-\frac{1}{2}\right)^{2}} \prod_{p \in \mathcal{P}} \frac{1}{1+\frac{1}{p(p-2)}} \\
= & \lim _{s \downarrow 1} \frac{1}{\zeta^{2}(s)} \frac{1-2 / 2^{s}}{\prod_{p \mid q_{1}}\left(1-2 / p^{s}\right) \prod_{p \mid q_{2}}\left(1-2 / p^{s}\right)} \mu\left(q_{1} q_{2}\right) \frac{1}{\left(1-\frac{1}{2}\right)^{2}} \prod_{p \in \mathcal{P}} \frac{1}{1+\frac{1}{p(p-2)}}=0,
\end{aligned}
$$

where we note that, since $\mu\left(q_{1} q_{2}\right)=0$ if $q_{1}$ and $q_{2}$ are even, we may assume $q_{1}$ or $q_{2}$ is odd. Therefore by letting $s \downarrow 1$ in (4.22), we obtain

$$
\sum_{n_{1}, n_{2}=1}^{\infty} \frac{\mu\left(n_{1} n_{2}\right)}{n_{1} n_{2}} c_{q_{1}}\left(n_{1}\right) c_{q_{2}}\left(n_{2}\right)=0,
$$

which is an extension of (3.12) to the case of two variables. Of course an extension of (3.13)

$$
\sum_{n_{1}, n_{2}=1}^{\infty} \frac{\lambda\left(n_{1} n_{2}\right)}{n_{1} n_{2}} c_{q_{1}}\left(n_{1}\right) c_{q_{2}}\left(n_{2}\right)=0
$$

clearly holds since $\lambda$ is completely multiplicative.

Next we consider extensions of Theorem 3.3 and Theorem 3.4. Ushiroya [Us] proved the following theorem which is an extension of Theorem 3.3.

Theorem 4.3. ([Us]) (i) Let $f\left(n_{1}, n_{2}\right)$ be an arithmetic function of two variables satisfying

$$
\sum_{n_{1}, n_{2}=1}^{\infty} 2^{\omega\left(n_{1}\right)} 2^{\omega\left(n_{2}\right)} \frac{\left|(f * \mu)\left(n_{1}, n_{2}\right)\right|}{n_{1} n_{2}}<\infty .
$$


Then its Ramanujan-Fourier series is pointwise convergent and

$$
f\left(n_{1}, n_{2}\right)=\sum_{q_{1}, q_{2}=1}^{\infty} a\left(q_{1}, q_{2}\right) c_{q_{1}}\left(n_{1}\right) c_{q_{2}}\left(n_{2}\right)
$$

holds where

$$
a\left(q_{1}, q_{2}\right)=\sum_{m_{1}, m_{2}=1}^{\infty} \frac{(f * \mu)\left(m_{1} q_{1}, m_{2} q_{2}\right)}{m_{1} q_{1} m_{2} q_{2}} .
$$

(ii) Let $f$ be a multiplicative function of two variables satisfying

$$
\sum_{p \in \mathcal{P}} \sum_{\substack{e_{1}, e_{2} \geqq 0 \\ e_{1}+e_{2} \geqq 1}} \frac{\left|(f * \mu)\left(p^{e_{1}}, p^{e_{2}}\right)\right|}{p^{e_{1}+e_{2}}}<\infty .
$$

Then its Ramanujan-Fourier series is pointwise convergent and

$$
f\left(n_{1}, n_{2}\right)=\sum_{q_{1}, q_{2}=1}^{\infty} a\left(q_{1}, q_{2}\right) c_{q_{1}}\left(n_{1}\right) c_{q_{2}}\left(n_{2}\right)
$$

holds where

$$
a\left(q_{1}, q_{2}\right)=\sum_{m_{1}, m_{2}=1}^{\infty} \frac{(f * \mu)\left(m_{1} q_{1}, m_{2} q_{2}\right)}{m_{1} q_{1} m_{2} q_{2}} .
$$

Moreover, if the mean value $M(f)=\lim _{x \rightarrow \infty} \sum_{n \leqq x} f(n)$ is not zero and if $\left\{q_{1}, q_{2}\right\}>1$, where $\left\{q_{1}, q_{2}\right\}$ denotes the least common multiple of $q_{1}$ and $q_{2}$, then $a\left(q_{1}, q_{2}\right)$ can be rewritten as

$$
\begin{aligned}
a\left(q_{1}, q_{2}\right) & =\prod_{p \in \mathcal{P}}\left(\sum_{e_{1}=\nu_{p}\left(q_{1}\right)} \sum_{e_{2}=\nu_{p}\left(q_{2}\right)} \frac{(f * \mu)\left(p^{e_{1}}, p^{e_{2}}\right)}{p^{e_{1}+e_{2}}}\right) \\
& =M(f) \prod_{p \mid\left\{q_{1}, q_{2}\right\}}\left\{\left(\sum_{e_{1}=\nu_{p}\left(q_{1}\right)} \sum_{e_{2}=\nu_{p}\left(q_{2}\right)} \frac{(f * \mu)\left(p^{e_{1}}, p^{e_{2}}\right)}{p^{e_{1}+e_{2}}}\right) /\left(\sum_{e_{1}=0} \sum_{e_{2}=0} \frac{(f * \mu)\left(p^{e_{1}}, p^{e_{2}}\right)}{p^{e_{1}+e_{2}}}\right)\right\} .
\end{aligned}
$$

We remark that many examples of the form

$$
f\left(n_{1}, n_{2}\right)=\sum_{q_{1}, q_{2}=1}^{\infty} a\left(q_{1}, q_{2}\right) c_{q_{1}}\left(n_{1}\right) c_{q_{2}}\left(n_{2}\right)
$$

are obtained in [Us].

Next we extend Theorem 3.4 to dual Ramanujan-Fourier series.

Theorem 4.4. Let $f$ be an arithmetic function of two variables satisfying

$$
\sum_{q_{1}, q_{2}=1}^{\infty} \frac{\left|(f * \mathbf{1})\left(q_{1}, q_{2}\right)\right|}{q_{1} q_{2}} \tau\left(q_{1}\right) \tau\left(q_{2}\right)<\infty .
$$

Then its dual Ramanujan-Fourier series is pointwise convergent and

$$
f\left(q_{1}, q_{2}\right)=\sum_{n_{1}, n_{2}=1}^{\infty} a\left(n_{1}, n_{2}\right) c_{q_{1}}\left(n_{1}\right) c_{q_{2}}\left(n_{2}\right)
$$

holds where

$$
a\left(n_{1}, n_{2}\right)=\sum_{m_{1}, m_{2}=1}^{\infty} \frac{(f * \mathbf{1})\left(n_{1} m_{1}, n_{2} m_{2}\right)}{n_{1} m_{1} n_{2} m_{2}} \mu\left(m_{1}, m_{2}\right) .
$$


Remark 4.2. Let $f$ be a multiplicative function of two variables satisfying

$$
\sum_{p \in \mathcal{P}} \sum_{\substack{e_{1}, e_{2} \geqq 0 \\ e_{1}+e_{2} \geqq 1}} \frac{\left|(f * \mathbf{1})\left(p^{e_{1}}, p^{e_{2}}\right)\right|}{p^{e_{1}+e_{2}}}\left(e_{1}+1\right)\left(e_{2}+1\right)<\infty .
$$

Then its dual Ramanujan-Fourier series is pointwise convergent and

$$
f\left(q_{1}, q_{2}\right)=\sum_{n_{1}, n_{2}=1}^{\infty} a\left(n_{1}, n_{2}\right) c_{q_{1}}\left(n_{1}\right) c_{q_{2}}\left(n_{2}\right)
$$

holds where

$$
a\left(n_{1}, n_{2}\right)=\sum_{m_{1}, m_{2}=1}^{\infty} \frac{(f * \mathbf{1})\left(n_{1} m_{1}, n_{2} m_{2}\right)}{n_{1} m_{1} n_{2} m_{2}} \mu\left(m_{1}, m_{2}\right) .
$$

Moreover, $a\left(n_{1}, n_{2}\right)$ can be rewritten as

$$
\begin{aligned}
a\left(n_{1}, n_{2}\right)=\prod_{p \in \mathcal{P}}( & \frac{(f * \mathbf{1})\left(p^{\nu_{p}\left(n_{1}\right)}, p^{\nu_{p}\left(n_{2}\right)}\right)}{p^{\nu_{p}\left(n_{1}\right)+\nu_{p}\left(n_{2}\right)}}-\frac{(f * \mathbf{1})\left(p^{\nu_{p}\left(n_{1}\right)+1}, p^{\nu_{p}\left(n_{2}\right)}\right)}{p^{\nu_{p}\left(n_{1}\right)+\nu_{p}\left(n_{2}\right)+1}} \\
& \left.-\frac{(f * \mathbf{1})\left(p^{\nu_{p}\left(n_{1}\right)}, p^{\nu_{p}\left(n_{2}\right)+1}\right)}{p^{\nu_{p}\left(n_{1}\right)+\nu_{p}\left(n_{2}\right)+1}}+\frac{(f * \mathbf{1})\left(p^{\nu_{p}\left(n_{1}\right)+1}, p^{\nu_{p}\left(n_{1}\right)+2}\right)}{p^{\nu_{p}\left(n_{1}\right)+\nu_{p}\left(n_{2}\right)+2}}\right) .
\end{aligned}
$$

Proof of Theorem 4.4. We proceed along the same lines as the proof of Theorem 3.4. We first see that $A\left(q_{1}, q_{2}\right)=q_{1} q_{2} \sum_{k_{1}, k_{2}=1}^{\infty} a\left(k_{1} q_{1}, k_{2} q_{2}\right)$ converges since

$$
\begin{aligned}
\sum_{\substack{k_{1} \leqq x \\
k_{2} \leqq y}}\left|a\left(k_{1} q_{1}, k_{2} q_{2}\right)\right| & \leqq \sum_{\substack{k_{1} \leqq x \\
k_{2} \leqq y}} \sum_{m_{1}, m_{2}=1}^{\infty} \frac{\left|(f * \mathbf{1})\left(k_{1} q_{1} m_{1}, k_{2} q_{2} m_{2}\right)\right|}{k_{1} q_{1} m_{1} k_{2} q_{2} m_{2}}\left|\mu\left(m_{1}, m_{2}\right)\right| \\
& \leqq \sum_{\ell_{1}, \ell_{2}=1}^{\infty} \frac{\left|(f * \mathbf{1})\left(\ell_{1}, \ell_{2}\right)\right|}{\ell_{1} \ell_{2}} \sum_{\substack{k_{1} \leqq x, k_{1}\left|\ell_{1} \\
k_{2} \leqq y, k_{2}\right| \ell_{2}}} 1 \\
& \leqq \sum_{\ell_{1}, \ell_{2}=1}^{\infty} \frac{\left|(f * \mathbf{1})\left(\ell_{1}, \ell_{2}\right)\right|}{\ell_{1} \ell_{2}} \tau\left(\ell_{1}\right) \tau\left(\ell_{2}\right)<\infty .
\end{aligned}
$$

Using Lemma 2.5 we can rewrite $A\left(q_{1}, q_{2}\right)$ as

$$
\begin{aligned}
A\left(q_{1}, q_{2}\right) & =q_{1} q_{2} \sum_{k_{1}, k_{2}=1}^{\infty} a\left(k_{1} q_{1}, k_{2} q_{2}\right)=\sum_{m_{1}, m_{2}=1}^{\infty} a\left(m_{1}, m_{2}\right) q_{1} I_{q_{1} \mid m_{1}} q_{2} I_{q_{2} \mid m_{2}} \\
& \left.=\sum_{m_{1}, m_{2}=1}^{\infty} a\left(m_{1}, m_{2}\right) \underset{\ell}{\left(\mathbf{1}_{\ell} c\right.}\right)\left(q_{1}, m_{1}\right)\left(\underset{\ell}{\mathbf{1}} \underset{\ell}{*}\left(q_{2}, m_{2}\right) .\right.
\end{aligned}
$$

From this we have

$$
\begin{aligned}
& \left.f\left(q_{1}, q_{2}\right)=(\mu * A)\left(q_{1}, q_{2}\right)=\sum_{m_{1}, m_{2}=1}^{\infty} a\left(m_{1}, m_{2}\right)\left(\mu \underset{\ell}{*} \underset{\ell}{*}{\underset{\ell}{\ell} c}^{*}\right)\right)\left(q_{1}, m_{1}\right)\left(\mu \underset{\ell}{*}\left(\mathbf{1}_{\ell}^{*} c\right)\right)\left(q_{2}, m_{2}\right) \\
& =\sum_{m_{1}, m_{2}=1}^{\infty} a\left(m_{1}, m_{2}\right)\left(\delta_{\ell}^{*} c\right)\left(q_{1}, m_{1}\right)\left(\delta_{\ell}^{*} c\right)\left(q_{2}, m_{2}\right) \\
& =\sum_{m_{1}, m_{2}=1}^{\infty} a\left(m_{1}, m_{2}\right) c_{q_{1}}\left(m_{1}\right) c_{q_{2}}\left(m_{2}\right) .
\end{aligned}
$$

This completes the proof of Theorem 4.4. 
Proof of Remark 4.2. We first note that, if $f$ is a multiplicative function of two variables, then $\left(q_{1}, q_{2}\right) \mapsto \frac{(f * \mathbf{1})\left(q_{1}, q_{2}\right)}{q_{1} q_{2}} \tau\left(q_{1}\right) \tau\left(q_{2}\right)$ is also a multiplicative function of two variables. Using $1+x \leqq \exp (x)$, we see that (4.24) follows from (4.26) since

$$
\begin{aligned}
\sum_{\substack{q_{1} \leqq Q_{1} \\
q_{2} \leqq Q_{2}}} \frac{\left|(f * \mathbf{1})\left(q_{1}, q_{2}\right)\right|}{q_{1} q_{2}} \tau\left(q_{1}\right) \tau\left(q_{2}\right) & \leqq \prod_{p \in \mathcal{P}}\left(1+\sum_{\substack{e_{1}, e_{2} \geqq 0 \\
e_{1}+e_{2} \geqq 1}} \frac{\left|(f * \mathbf{1})\left(p^{e_{1}}, p^{e_{2}}\right)\right|}{p^{e_{1}} p^{e_{2}}} \tau\left(p^{e_{1}}\right) \tau\left(p^{e_{2}}\right)\right) \\
& \leqq \prod_{p \in \mathcal{P}} \exp \left(\sum_{\substack{e_{1}, e_{2} \geqq 0 \\
e_{1}+e_{2} \geqq 1}} \frac{\left|(f * \mathbf{1})\left(p^{e_{1}}, p^{e_{2}}\right)\right|}{p^{e_{1}} p^{e_{2}}} \tau\left(p^{e_{1}}\right) \tau\left(p^{e_{2}}\right)\right) \\
& =\exp \left(\sum_{\substack{\mathcal{P}_{2} \\
e_{e_{1}, e_{2} \geqq 0}}} \sum_{e_{1}+e_{2} \geqq 1} \frac{\left|(f * \mathbf{1})\left(p^{e_{1}}, p^{e_{2}}\right)\right|}{p^{e_{1}+e_{2}}}\left(e_{1}+1\right)\left(e_{2}+1\right)\right)<\infty
\end{aligned}
$$

holds for any $Q_{1}, Q_{2}>1$. Therefore (4.25) holds by Theorem 4.4. In the expression of (4.25), we set, for $i=1,2, n_{i}=\prod p_{j}^{e_{i j}}, m_{i}=r_{i} \prod p_{j}^{d_{i j}}$ where $\left(r_{i}, n_{1} n_{2}\right)=1, e_{i j} \geqq 1$, and $d_{i j} \geqq 0$. Then we have

$$
a\left(n_{1}, n_{2}\right)=\sum_{\substack{d_{i j} \geqq 0, r_{i} \geqq 1 \\\left(r_{i}, n_{1} n_{2}\right)=1}} \frac{(f * \mathbf{1})\left(r_{1} \prod p_{j}^{d_{1 j}+e_{1 j}}, r_{2} \prod p_{j}^{d_{2 j}+e_{2 j}}\right)}{r_{1} r_{2} \prod p_{j}^{d_{1 j}+e_{1 j}+d_{2 j}+e_{2 j}}} \mu\left(r_{1} \prod p_{j}^{d_{1 j}}, r_{2} \prod p_{j}^{d_{2 j}}\right) .
$$

Since $f * 1$ is multiplicative and since $\mu\left(p_{j}^{d_{1 j}}, p_{j}^{d_{2 j}}\right)=0$ if $d_{1 j} \geqq 2$ or $d_{2 j} \geqq 2$ for some $j$, we obtain

$$
\begin{aligned}
& a\left(n_{1}, n_{2}\right)=\prod_{j}\left(\sum_{0 \leqq d_{i j} \leqq 1} \frac{(f * \mathbf{1})\left(p_{j}^{d_{1 j}+e_{1 j}}, p_{j}^{d_{2 j}+e_{2 j}}\right)}{p_{j}^{d_{1 j}+e_{1 j}+d_{2 j}+e_{2 j}}} \mu\left(p_{j}^{d_{1 j}}, p_{j}^{d_{2 j}}\right)\right) \times \sum_{\substack{r_{i} \geqq 1 \\
\left(r, n_{1} n_{2}\right)=1}} \frac{(f * \mathbf{1})\left(r_{1}, r_{2}\right)}{r_{1} r_{2}} \mu\left(r_{1}, r_{2}\right) \\
& =\prod_{p \mid n_{1} n_{2}}\left(\frac{(f * \mathbf{1})\left(p^{\nu_{p}\left(n_{1}\right)}, p^{\nu_{p}\left(n_{2}\right)}\right)}{p^{\nu_{p}\left(n_{1}\right)+\nu_{p}\left(n_{2}\right)}}-\frac{(f * \mathbf{1})\left(p^{\nu_{p}\left(n_{1}\right)+1}, p^{\nu_{p}\left(n_{2}\right)}\right)}{p^{\nu_{p}\left(n_{1}\right)+\nu_{p}\left(n_{2}\right)+1}}\right. \\
& \left.-\frac{(f * \mathbf{1})\left(p^{\nu_{p}\left(n_{1}\right)}, p^{\nu_{p}\left(n_{2}\right)+1}\right)}{p^{\nu_{p}\left(n_{1}\right)+\nu_{p}\left(n_{2}\right)+1}}+\frac{(f * \mathbf{1})\left(p^{\nu_{p}\left(n_{1}\right)+1}, p^{\nu_{p}\left(n_{1}\right)+2}\right)}{p^{\nu_{p}\left(n_{1}\right)+\nu_{p}\left(n_{2}\right)+2}}\right) \\
& \times \prod_{p \nmid n_{1} n_{2}}\left(1-\frac{f(p, 1)}{p}-\frac{f(1, p)}{p}+\frac{f(p, p)}{p^{2}}\right) \\
& =\prod_{p \in \mathcal{P}}\left(\frac{(f * \mathbf{1})\left(p^{\nu_{p}\left(n_{1}\right)}, p^{\nu_{p}\left(n_{2}\right)}\right)}{p^{\nu_{p}\left(n_{1}\right)+\nu_{p}\left(n_{2}\right)}}-\frac{(f * \mathbf{1})\left(p^{\nu_{p}\left(n_{1}\right)+1}, p^{\nu_{p}\left(n_{2}\right)}\right)}{p^{\nu_{p}\left(n_{1}\right)+\nu_{p}\left(n_{2}\right)+1}}\right. \\
& \left.-\frac{(f * \mathbf{1})\left(p^{\nu_{p}\left(n_{1}\right)}, p^{\nu_{p}\left(n_{2}\right)+1}\right)}{p^{\nu_{p}\left(n_{1}\right)+\nu_{p}\left(n_{2}\right)+1}}+\frac{(f * \mathbf{1})\left(p^{\nu_{p}\left(n_{1}\right)+1}, p^{\nu_{p}\left(n_{1}\right)+2}\right)}{p^{\nu_{p}\left(n_{1}\right)+\nu_{p}\left(n_{2}\right)+2}}\right),
\end{aligned}
$$

which completes the proof of Remark 4.2 .

If we take $f=\mu$ in Theorem 3.4, then it is obvious that

$$
\mu(q)=\sum_{n=1}^{\infty} a(n) c_{q}(n)
$$

holds where $a(n)=\delta(n)$. The following example is an extension of the above trivial example. 
Example 4.2. We have

$$
\mu\left(q_{1} q_{2}\right)=\frac{1}{\zeta(2)} \sum_{n_{1}, n_{2}=1}^{\infty} \frac{\mu\left(K\left(\left(n_{1}, n_{2}\right)\right)\right) \varphi\left(K\left(\left(n_{1}, n_{2}\right)\right)\right)}{n_{1} n_{2} \psi\left(K\left(n_{1} n_{2}\right)\right)} c_{q_{1}}\left(n_{1}\right) c_{q_{2}}\left(n_{2}\right) .
$$

Proof. Let $f\left(q_{1}, q_{2}\right)=\mu\left(q_{1} q_{2}\right)$. Then it is easy to see that

$$
\begin{aligned}
& (f * \mathbf{1})\left(p^{k}, 1\right)=(f * \mathbf{1})\left(1, p^{k}\right)=0 \quad \text { if } k \geqq 1, \\
& (f * \mathbf{1})\left(p^{k}, p^{\ell}\right)=-1 \quad \text { if } k, \ell \geqq 1 .
\end{aligned}
$$

From this we see that (4.26) holds. We have

$$
\begin{aligned}
& \frac{(f * \mathbf{1})\left(p^{k}, p^{\ell}\right)}{p^{k+\ell}}-\frac{(f * \mathbf{1})\left(p^{k+1}, p^{\ell}\right)}{p^{k+\ell+1}}-\frac{(f * \mathbf{1})\left(p^{k}, p^{\ell+1}\right)}{p^{k+\ell+1}}+\frac{(f * \mathbf{1})\left(p^{k+1}, p^{\ell+1}\right)}{p^{k+\ell+2}} \\
& = \begin{cases}1-1 / p^{2} & \text { if } k=\ell=0 \\
1 / p^{\ell+1}-1 / p^{\ell+2} & \text { if } k=0, \quad \ell \geqq 1 \\
1 / p^{k+1}-1 / p^{k+2} & \text { if } k \geqq 1, \quad \ell=0 \\
-1 / p^{k+\ell}+2 / p^{k+\ell+1}-1 / p^{k+\ell+2} & \text { if } k, \ell \geqq 1 .\end{cases}
\end{aligned}
$$

Therefore we have by Remark 4.2

$$
\begin{aligned}
& a\left(n_{1}, n_{2}\right)=\prod_{p \nmid n_{1} n_{2}}\left(1-\frac{1}{p^{2}}\right) \prod_{\substack{p \nmid n_{1} \\
p \mid n_{2}}}\left(\frac{1}{p^{\nu_{p}\left(n_{2}\right)+1}}-\frac{1}{p^{\nu_{p}\left(n_{2}\right)+2}}\right) \prod_{\substack{p \mid n_{1} \\
p \nmid n_{2}}}\left(\frac{1}{p^{\nu_{p}\left(n_{1}\right)+1}}-\frac{1}{p^{\nu_{p}\left(n_{1}\right)+2}}\right) \\
& \times \prod_{p \mid n_{1}}\left(-\frac{1}{p^{\nu_{p}\left(n_{1}\right)+\nu_{p}\left(n_{2}\right)}}+\frac{2}{p^{\nu_{p}\left(n_{1}\right)+\nu_{p}\left(n_{2}\right)+1}}-\frac{1}{p^{\nu_{p}\left(n_{1}\right)+\nu_{p}\left(n_{2}\right)+2}}\right) \\
& =\prod_{p \nmid n_{1} n_{2}}\left(1-\frac{1}{p^{2}}\right) \prod_{\substack{p \nmid n_{1} \\
p \mid n_{2}}} \frac{1}{p^{\nu_{p}\left(n_{2}\right)+1}}\left(1-\frac{1}{p}\right) \prod_{\substack{p \mid n_{1} \\
p \nmid n_{2}}} \frac{1}{p^{\nu_{p}\left(n_{1}\right)+1}}\left(1-\frac{1}{p}\right) \\
& \times \prod_{\substack{p\left|n_{1} \\
p\right| n_{2}}} \frac{-1}{p^{\nu_{p}\left(n_{1}\right)+\nu_{p}\left(n_{2}\right)}}\left(1-\frac{2}{p}+\frac{1}{p^{2}}\right) \\
& =\prod_{p \in \mathcal{P}}\left(1-\frac{1}{p^{2}}\right) \prod_{\substack{p \nmid n_{1} \\
p \mid n_{2}}} \frac{1}{p^{\nu p}\left(n_{2}\right)+1} \frac{1-1 / p}{1-1 / p^{2}} \prod_{\substack{p \mid n_{1} \\
p \nmid n_{2}}} \frac{1}{p^{\nu_{p}\left(n_{1}\right)+1}} \frac{1-1 / p}{1-1 / p^{2}} \prod_{\substack{p\left|n_{1} \\
p\right| n_{2}}} \frac{-1}{p^{\nu_{p}\left(n_{1}\right)+\nu_{p}\left(n_{2}\right)}} \frac{(1-1 / p)^{2}}{1-1 / p^{2}} \\
& =\frac{1}{\zeta(2)} \prod_{\substack{p \nmid n_{1} \\
p \mid n_{2}}} \frac{1}{p^{\nu_{p}\left(n_{2}\right)+1}} \frac{1}{1+1 / p} \prod_{\substack{p \mid n_{1} \\
p \nmid n_{2}}} \frac{1}{p^{\nu_{p}\left(n_{1}\right)+1}} \frac{1}{1+1 / p} \prod_{p \mid\left(n_{1}, n_{2}\right)} \frac{-1}{p^{\nu_{p}\left(n_{1}\right)+\nu_{p}\left(n_{2}\right)}} \frac{1-1 / p}{1+1 / p} \\
& =\frac{1}{\zeta(2)} \prod_{p \mid n_{1} n_{2}} \frac{1}{p^{\nu_{p}\left(n_{1}\right)+\nu_{p}\left(n_{2}\right)}} \frac{1}{p(1+1 / p)} \prod_{p \mid\left(n_{1}, n_{2}\right)}(-1) p(1-1 / p) \\
& =\frac{1}{\zeta(2)} \frac{\mu\left(K\left(\left(n_{1}, n_{2}\right)\right)\right) \varphi\left(K\left(\left(n_{1}, n_{2}\right)\right)\right)}{n_{1} n_{2} \psi\left(K\left(n_{1} n_{2}\right)\right)},
\end{aligned}
$$

which completes the proof of Example 4.2.

\section{Acknowledgment.}

The author sincerely thanks the referees, whose comments and suggestions essentially improved this paper. 


\section{References}

[De] H. Delange, On Ramanujan expansions of certain arithmetical functions, Acta. Arithmetica, 31 (1976), $259-270$.

[Ha] G.H. Hardy, Note on Ramanujan's trigonometrical function $c_{q}(n)$, and certain series of arithmetical functions, Proc. Cambridge Phil. Soc., 20 (1921), 263-271.

[Jo] K.R. Johnson, Reciprocity in Ramanujan's sum, Math. Mag., 59 (1986), 216-222.

[Lu] L. G. Lucht, A survey of Ramanujan expansions, International Journal of Number Theory, 6 (2010), $1785-1799$.

[Rm] M. Ram Murty, Ramanujan series for arithmetical functions, Hardy-Ramanujan Journal, 36 (2013), $21-33$.

[Ra] S. Ramanujan, On certain trigonometrical sums and their applications in the theory of numbers, Transactions of the Cambridge Phil. Society, 22 (1918), 179-199.

[SS] W. Schwarz and J. Spilker, Arithmetical Functions, Cambridge Univ. Press, 1994.

[Si] R. Sivaramakrishnan, Classical Theory of Arithmetic Functions, Marcel Dekker, 1989.

[To] L. Tóth, Multiplicative Arithmetic Functions of Several Variables, a Survey, in vol. Mathematics Without Boundaries, Surveys in Pure Mathematics, Th. M. Rassias, P. Pardalos (Editors), Springer, New York, $2014,483-514$.

[Us] N. Ushiroya, Ramanujan-Fourier series of arithmetic functions of two variables, Hardy-Ramanujan Journal, 39 (2016), 1-20.

Noboru Ushiroya

National Institute of Technology, Wakayama College, 77 Noshima Nada Gobo Wakayama, Japan

e-mail: ushiroya@wakayama-nct.ac.jp 